IASSNS-HEP-96-44

UFIFT-HEP-96-12

hep-ph/9605325

May 1996

\title{
Stable Superstring Relics
}

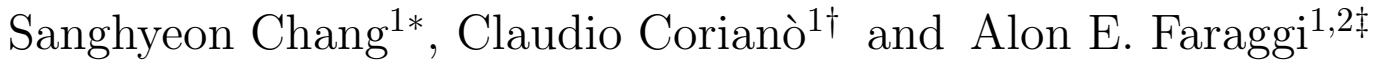 \\ ${ }^{1}$ Institute for Fundamental Theory, Department of Physics, \\ University of Florida, Gainesville, FL 32611, USA \\ 2 School of Natural Sciences, Institute for Advanced Study \\ Olden Lane, Princeton, NJ 08540 USA
}

\begin{abstract}
We investigate the cosmological constraints on exotic stable matter states which arise in realistic free fermionic superstring models. These states appear in the superstring models due to a "Wilson-line" breaking of the unifying nonAbelian gauge symmetry. In the models that we consider the unifying $S O(10)$ gauge symmetry is broken at the string level to $S O(6) \times S O(4), S U(5) \times U(1)$ or $S U(3) \times S U(2) \times U(1)^{2}$. The exotic matter states are classified according to the patterns of the $S O(10)$ symmetry breaking. In $S O(6) \times S O(4)$ and $S U(5) \times U(1)$ type models one obtains fractionally charged states with $Q_{\text {e.m. }}=$ $\pm 1 / 2$. In $S U(3) \times S U(2) \times U(1)^{2}$ type models one also obtains states with the regular charges under the Standard Model gauge group but with "fractional" charges under the $U(1)_{Z^{\prime}}$ symmetry. These states include down-like color triplets and electroweak doublets, as well as states which are Standard Model singlets. By analyzing the renormalizable and nonrenormalizable terms of the superpotential in a specific superstring model, we show that these exotic states can be stable. We investigate the cosmological constraints on the masses and relic density of the exotic states. We propose that, while the abundance and the masses of the fractionally charged states are highly constrained, the Standard Model - like states, and in particular the Standard Model singlet, are good dark matter candidates.
\end{abstract}

\footnotetext{
* E-mail address: schang@phys.ufl.edu

$\dagger$ E-mail address: coriano@phys.ufl.edu

¥ E-mail address: faraggi@phys.ufl.edu

$\S$ Permanent address.
} 


\section{Introduction}

Superstring theories [1] are believed to provide a consistent framework for the unification of gravity with the gauge interactions. An important task is to connect superstring theory with the Standard Model [2, 3]. Several approaches may be pursued to derive the Standard Model from superstring theory. One possibility is to go through a simple [4] or a semi-simple [5, 6, 7, 8, 9] unifying gauge group at intermediate energy scale. Another is to derive the Standard Model directly from superstring theory [10, 11, 12, 13, 14]. Proton lifetime considerations motivate the hypothesis that the Standard Model must be obtained directly from superstring theory [15, 16]. A second important question is whether there exist some property of superstring models that will distinguish them from other attempts to understand the origin of the Standard Model. If such a property exists it may result in an experimental signal that can prove or disprove the validity of superstring unification.

In this paper we explore one such possible signature of superstring unification. We argue that realistic superstring models produce additional heavy stable matter, beyond the spectrum of the Standard Model. The specific matter states and their properties vary between models. However, the existence of additional stable matter, beyond the observed spectrum of the Standard Model, is generic. One type of such generic states in superstring models are of course the moduli fields. Indeed, it as been argued that because of the absence of superpotential for the moduli fields, they will decouple at a very early stage in the evolution of the universe and will overclose the universe [17]. However, in the class of models that we study, it has been suggested that all the moduli (except, of course, the dilaton) are projected out by the GSO projections [18]. Thus, these models the cosmological moduli problem can be resolved. The matter states that we study in this paper arise due to the superstringy breaking of the unifying gauge symmetry. We investigate the possibility that these stringy stable matter states can be the dark matter and can perhaps be detected.

In the attempts to derive the Standard Model from superstring theory one traditionally starts with a larger, unifying, gauge symmetry $G$. The gauge symmetry is then broken to the Standard Model by means of Wilson lines. In many respects the unifying gauge symmetry $G$ is similar to the gauge group of four dimensional grand unification and the Wilson lines are similar to the Higgs bosons in the adjoint representation. However, there are some notable differences. The eigenvalues of the Wilson lines are quantized while the eigenvalues of the Higgs in the adjoint representation are continuous. Another important difference is that the breaking of the gauge symmetries by Wilson lines results in massless states that do not fit into multiplets of the original unbroken gauge symmetry. We refer to such states generically as exotic "Wilsonian" matter states. This is an important property as it may result in conserved quantum numbers that will indicate the stability of these massless "Wilsonian" states. The simplest example of this phenomenon is the existence of states with fractional electric charge in the massless spectrum of superstring models 
[19, 20, 21, 22]. Such states are stable due to electric charge conservation. As there exist strong constraints on their masses and abundance, states with fractional electric charge must be diluted away or extremely massive. Remarkably, however, the same "Wilson line" breaking mechanism, which produces matter with fractional electric charge, is also responsible for the existence of states which carry the "standard" charges under the Standard Model gauge group but which carry fractional charges under a different subgroup of the unifying gauge group. For example, if the group $G$ is $S O(10)$ then the "Wilsonian" states carry non-standard charges under the $U(1)_{Z^{\prime}}$ symmetry, which is embedded in $S O(10)$ and is orthogonal to $U(1)_{Y}$. Such states can therefore be stable if the $U(1)_{Z^{\prime}}$ gauge symmetry remains unbroken down to low energies, or if some residual local discrete symmetry is left unbroken after the $U(1)_{Z^{\prime}}$ symmetry breaking.

In this paper we propose that the existence of heavy stable "Wilsonian" matter may be the "smoking gun" of string unification. The existence of stable "Wilsonian" states at intermediate energy scale have important cosmological implications. In a previous letter [24] we examined the possibility that one type of the extra "Wilsonian" states constitute the dark matter of the universe. These states consist of heavy downlike quark with the standard down-like charge assignment. Due to its role in the string unification we referred to this type of particle as the uniton. We proposed that because of its "fractional" charge under the $U(1)_{Z^{\prime}}$ symmetry, the uniton may be stable.

In this paper we extend the analysis of ref. [24]. We discuss in detail the cosmological constraints on the existence of heavy "Wilsonian" states. We provide the details of the analysis of ref. [24] and extend our investigation to other exotic matter states which appear in the realistic superstring derived models. In the superstring models that we consider the unifying gauge symmetry is $S O(10)$. The $S O(10)$ symmetry is broken at the string level to $S O(6) \times S O(4), S U(5) \times U(1)$ or $S U(3) \times S U(2) \times U(1)^{2}$. We classify the exotic "Wilsonian" matter states according to the pattern of the $S O(10)$ symmetry breaking. The $S O(6) \times S O(4)$ and $S U(5) \times U(1)$ type models give rise to fractionally charged states with $Q_{\text {e.m. }}= \pm 1 / 2$. On the other hand, the $S U(3) \times S U(2) \times U(1)^{2}$ type models produce in addition states with the regular charges under the Standard Model gauge group but with "fractional" charges under the $U(1)_{Z^{\prime}}$ gauge group. These states include down-like color triplets and electroweak doublets, as well as states which are Standard Model singlets. We show, by analyzing the renormalizable and nonrenormalizable terms of the superpotential in a specific superstring model, that these exotic states can be stable. We investigate the cosmological constraints on the masses and relic density of the exotic states. We propose that, while the abundance and the masses of the fractionally charged states are highly constrained, the Standard Model - like states, and in particular the Standard Model singlet, are possible candidates for the dark matter.

The exotic "Wilsonian" matter states that we study in this paper are divided into three distinct classes: 
The first class consists of down-like color triplets with "fractional" charge under the $U(1)_{Z^{\prime}}$ symmetry. The existence of such a heavy colored state is motivated from the constraints arising from string gauge coupling unification [25]. In a specific superstring model we analyze the interaction terms of this colored triplets with the Standard Model states. In that model we show that if we assume that an hidden $S U(3)_{H}$ gauge group remains unbroken then all the interaction terms in the superpotential vanish to any order of nonrenormalizable terms. This result arises due to the fractional $U(1)_{Z^{\prime}}$ of the color "Wilsonian" triplets and because in the specific string model which we analyze in detail the only other Standard Model singlet states which carry fractional $U(1)_{Z^{\prime}}$ charge are triplets of $S U(3)_{H}$. Thus, we argue that the "Wilsonian" states can arise as stable states. We then proceed to analyze the constraints on the relic density of stable heavy color triplets. The heavy color triplets can annihilate into quarks, squarks, gluons and gluinos. We examine the possibility that the heavy color down-like triplets are the dark matter. The heavy stable down-like states form charged and neutral meson bound states with the up and down quarks, respectively. An important issue in this regard is the mass splitting between the charged and neutral meson states. We argue that with our present understanding of QCD, and the experimental determination of the light quark masses, there exists a region in the parameter space in which the neutral heavy meson state is the neutral one.

Next we examine the constraints on the relic density of fractionally charged states, with electric charge $\pm 1 / 2$. We show that generically these states either have to be super massive or have to be inflated away. We demonstrate in one specific model that all the fractionally charged states have a cubic level mass term. Thus, all the fractionally charged states can decouple from the massless spectrum by some choices of flat directions. An alternative is that all the fractionally charged states are confined by some non-Abelian gauge group in the hidden sector. Another novel feature that arises in some string models is the appearance of fractionally charged baryons and fractionally charged leptons. Thus, one can speculate that the these baryons and leptons will continue to scatter in the early universe until they coalesce to form neutral heavy hydrogen-like atoms.

The final class of "Wilsonian" states that we consider are Standard Model singlets with fractional $U(1)_{Z^{\prime}}$ charge. These type of states arise in the superstring derived standard-like models and interact with the Standard Model states only via the $U(1)_{Z^{\prime}}$ gauge boson and are candidates for weakly interacting dark matter (WIMPs). We examine four possible scenarios: with and without inflation and with the $U(1)_{Z^{\prime}}$ gauge boson being heavier or lighter than the "Wilsonian"-singlet states. We propose that this Standard Model singlet states is the most likely candidate for the dark matter in the superstring models.

Our paper is organized as follows. In section two we review the realistic free fermionic superstring models. In section three we describe the exotic "Wilsonian" states and classify them according to the patterns of the $S O(10)$ symmetry break- 
ing. In section four we examine the cosmological constraints on the different classes of "Wilsonian" matter states which are obtained from the superstring models. In section five we present our conclusions. Our discussion of the mass difference in the heavy meson system are given in appendix A. The details of the calculation of the annihilation cross sections are give in appendix B.

\section{Realistic free fermionic models}

In the free fermionic formulation of the heterotic string [2] all the degrees of freedom needed to cancel the conformal anomaly are represented in terms of internal free fermions propagating on the string world-sheet. In four dimensions, this requires 20 left-moving and 44 right-moving real world-sheet fermions. Equivalently, some real fermions may be paired to form complex fermions. Under parallel transport around a noncontractible loop, the fermionic states pick up a phase. Specification of the phases for all world-sheet fermions around all noncontractible loops contributes to the spin structure of the model. Such spin structures are usually given in the form of boundary condition "vectors", with each element of the vector specifying the phase of a corresponding world-sheet fermion. The possible spin structures are constrained by string consistency requirements (e.g. modular invariance). A model is constructed by choosing a set of boundary condition basis vectors, which satisfies the modular invariance constraints. The basis vectors, $b_{k}$, span a finite additive group $\Xi=\sum_{k} n_{k} b_{k}$ where $n_{k}=0, \cdots, N_{z_{k}}-1$. The physical massless states in the Hilbert space of a given sector $\alpha \in \Xi$, are obtained by acting on the vacuum with bosonic and fermionic operators and by applying the generalized GSO projections. The $U(1)$ charges, $Q(f)$, with respect to the unbroken Cartan generators of the four dimensional gauge group, which are in one-to-one correspondence with the $U(1)$ currents $f^{*} f$ for each complex fermion $f$, are given by:

$$
Q(f)=\frac{1}{2} \alpha(f)+F(f)
$$

where $\alpha(f)$ is the boundary condition of the world-sheet fermion $f$ in the sector $\alpha$, and $F_{\alpha}(f)$ is a fermion number operator counting each mode of $f$ once (and if $f$ is complex, $f^{*}$ minus once). For periodic fermions, $\alpha(f)=1$, the vacuum is a spinor in order to represent the Clifford algebra of the corresponding zero modes. For each periodic complex fermion $f$ there are two degenerate vacua $|+\rangle,|-\rangle$, annihilated by the zero modes $f_{0}$ and $f_{0}{ }^{*}$ and with fermion numbers $F(f)=0,-1$, respectively.

The realistic models in the free fermionic formulation are generated by a basis of boundary condition vectors for all world-sheet fermions [6, 11, 17, 12, 23, 13, 9, 14]. The basis is constructed in two stages. The first stage consists of the NAHE set [6, 13], which is a set of five boundary condition basis vectors, $\left\{\mathbf{1}, S, b_{1}, b_{2}, b_{3}\right\}$. The gauge group after the NAHE set is $S O(10) \times S O(6)^{3} \times E_{8}$ with $N=1$ space-time supersymmetry. The vector $S$ is the supersymmetry generator and the superpartners of the states from a given sector $\alpha$ are obtained from the sector $S+\alpha$. The space-time 
vector bosons that generate the gauge group arise from the Neveu-Schwarz sector and from the sector $\xi=1+b_{1}+b_{2}+b_{3}$. The Neveu-Schwarz sector produces the generators of $S O(10) \times S O(6)^{3} \times S O(16)$. The sector $\xi=1+b_{1}+b_{2}+b_{3}$ produces the spinorial 128 of $S O(16)$ and completes the hidden gauge group to $E_{8}$. The vectors $b_{1}, b_{2}$ and $b_{3}$ correspond to the three twisted sectors in the corresponding orbifold formulation and produce 48 spinorial 16 of $S O(10)$, sixteen from each sector $b_{1}, b_{2}$ and $b_{3}$.

The NAHE set divides the 44 right-moving and 20 left-moving real internal fermions in the following way: $\bar{\psi}^{1, \cdots, 5}$ are complex and produce the observable $S O(10)$ symmetry; $\bar{\phi}^{1, \cdots, 8}$ are complex and produce the hidden $E_{8}$ gauge group; $\left\{\bar{\eta}^{1}, \bar{y}^{3, \cdots, 6}\right\}$, $\left\{\bar{\eta}^{2}, \bar{y}^{1,2}, \bar{\omega}^{5,6}\right\},\left\{\bar{\eta}^{3}, \bar{\omega}^{1, \cdots, 4}\right\}$ give rise to the three horizontal $S O(6)$ symmetries. The left-moving $\{y, \omega\}$ states are divided to, $\left\{y^{3, \cdots, 6}\right\},\left\{y^{1,2}, \omega^{5,6}\right\},\left\{\omega^{1, \cdots, 4}\right\}$. The leftmoving $\chi^{12}, \chi^{34}, \chi^{56}$ states carry the supersymmetry charges. Each sector $b_{1}, b_{2}$ and $b_{3}$ carries periodic boundary conditions under $\left(\psi^{\mu} \mid \bar{\psi}^{1, \cdots, 5}\right)$ and one of the three groups: $\left(\chi_{12},\left\{y^{3, \cdots, 6} \mid \bar{y}^{3, \cdots 6}\right\}, \bar{\eta}^{1}\right),\left(\chi_{34},\left\{y^{1,2}, \omega^{5,6} \mid \bar{y}^{1,2} \bar{\omega}^{5,6}\right\}, \bar{\eta}^{2}\right)$ and $\left(\chi_{56},\left\{\omega^{1, \cdots, 4} \mid \bar{\omega}^{1, \cdots 4}\right\}, \bar{\eta}^{3}\right)$. The division of the internal fermions is a reflection of the underlying $Z_{2} \times Z_{2}$ orbifold compactification [26]. The set of internal fermions $\{y, \omega \mid \bar{y}, \bar{\omega}\}^{1, \cdots, 6}$ corresponds to the left-right symmetric conformal field theory of the heterotic string, or to the six dimensional compactified manifold in a bosonic formulation.

The second stage of the basis construction consists of adding three additional basis vectors to the NAHE set. The allowed boundary conditions in the additional basis vectors are constrained by the string consistency constraints, i.e. modular invariance and world-sheet supersymmetry. These three additional basis vectors correspond to "Wilson lines" in the orbifold formulation. The additional basis vectors distinguish between different models and determine their low energy properties. Three additional vectors are needed to reduce the number of generations to three, one from each sector $b_{1}, b_{2}$ and $b_{3}$. At the same time the additional boundary condition basis vectors break the gauge symmetries of the NAHE set. The $S O(10)$ symmetry is broken to one of its subgroups $S U(5) \times U(1), S O(6) \times S O(4)$ or $S U(3) \times S U(2) \times U(1)_{B-L} \times U(1)_{T_{3_{R}}}$. This is achieved by the assignment of boundary conditions to the set $\bar{\psi}^{1, \cdots, 5}$ :

$$
\begin{gathered}
b\left\{\bar{\psi}_{\frac{1}{2}}^{1 \cdots 5}\right\}=\left\{\frac{1}{2} \frac{1}{2} \frac{1}{2} \frac{1}{2} \frac{1}{2}\right\} \Rightarrow S U(5) \times U(1), \\
b\left\{\bar{\psi}_{\frac{1}{2}}^{1 \cdots 5}\right\}=\{11100\} \Rightarrow S O(6) \times S O(4) .
\end{gathered}
$$

To break the $S O(10)$ symmetry to $S U(3) \times S U(2) \times U(1)_{C} \times U(1)_{L}$ 常 both steps, (2.2) and (2.3), are used, in two separate basis vectors. In the superstring derived standard-like models the three additional basis vectors, beyond the NAHE set, are denoted by $\{\alpha, \beta, \gamma\}$. The two basis vectors $\alpha$ and $\beta$ break the $S O(10)$ symmetry to $S O(6) \times S O(4)$ and the vector $\gamma$ breaks the $S O(10)$ symmetry to $S U(5) \times U(1)$. Since the standard-like superstring derived models contain the $S O(6) \times S O(4)$ breaking

\footnotetext{
${ }^{*} U(1)_{C}=\frac{3}{2} U(1)_{B-L} ; U(1)_{L}=2 U(1)_{T_{3}}$.
} 
sectors, as well as the $S U(5) \times U(1)$ breaking sectors, their massless spectra admits also the exotic representations that can appear in these models. Therefore, below we will focus mostly on the superstring derived standard-like models and comment on the overlap with the other models.

The observable gauge group after application of the generalized GSO projections is $S U(3)_{C} \times U(1)_{C} \times S U(2)_{L} \times U(1)_{L} \times U(1)^{3} \times U(1)^{n}$. The electromagnetic charge is given by

$$
U(1)_{\mathrm{e} . \mathrm{m} .}=T_{3_{L}}+U(1)_{Y}
$$

where $T_{3_{L}}$ is the diagonal generator of $S U(2)_{L}$ and $U(1)_{Y}$ is the weak hypercharge. The weak hypercharge is given $b^{\dagger}$

$$
U(1)_{Y}=\frac{1}{3} U(1)_{C}+\frac{1}{2} U(1)_{L}
$$

and the orthogonal combination is given by

$$
U(1)_{Z^{\prime}}=U(1)_{C}-U(1)_{L}
$$

The horizontal $S O(6)^{3}$ symmetries are broken to factors of $U(1)$ s. The first three horizontal $U(1)$ symmetries arise from the world-sheet complex fermions, $\bar{\eta}^{j}, \quad(j=$ $1,2,3)$. The additional $U(1)^{n}$ symmetries arise from complexifying two right-moving real fermions from the set $\{\bar{y}, \bar{\omega}\}^{1, \cdots, 6}$. For each right-moving gauged $U(1)$ symmetry there is a corresponding left-moving global $U(1)$ symmetry. Alternatively, a leftmoving real fermion can be paired with a right-moving real fermion to form an Ising model operator 28 . The hidden gauge group after application of the generalized GSO projections is $S U(5)_{H} \times S U(3)_{H} \times U(1)^{2}$. The $U(1)$ symmetries in the hidden sector, $U(1)_{7}$ and $U(1)_{8}$, correspond to the world-sheet currents $\bar{\phi}^{1} \bar{\phi}^{1^{*}}-\bar{\phi}^{8} \bar{\phi}^{8^{*}}$ and $-2 \bar{\phi}^{j} \bar{\phi}^{j^{*}}+\bar{\phi}^{1} \bar{\phi}^{1^{*}}+4 \bar{\phi}^{2} \bar{\phi}^{2^{*}}+\bar{\phi}^{8} \bar{\phi}^{8^{*}}$ respectively, where summation on $j=5, \cdots, 7$ is implied.

The massless spectrum of the standard-like models contains three chiral generations from the sectors $b_{1}, b_{2}$ and $b_{3}$ with charges under the horizontal symmetries. Three generations from the sectors $b_{1}, b_{2}$ and $b_{3}$ are common to all the free fermionic standard-like models. For example in the model of Ref. [27] we have,

$$
\begin{aligned}
& \left(e_{L}^{c}+u_{L}^{c}\right)_{\frac{1}{2}, 0,0, \frac{1}{2}, 0,0}+\left(d_{L}^{c}+N_{L}^{c}\right)_{\frac{1}{2}, 0,0, \frac{1}{2}, 0,0}+(L)_{\frac{1}{2}, 0,0,-\frac{1}{2}, 0,0}+(Q)_{\frac{1}{2}, 0,0,-\frac{1}{2}, 0,0}, \\
& \left(e_{L}^{c}+u_{L}^{c}\right)_{0, \frac{1}{2}, 0,0, \frac{1}{2}, 0}+\left(N_{L}^{c}+d_{L}^{c}\right)_{0, \frac{1}{2}, 0,0, \frac{1}{2}, 0}+(L)_{0, \frac{1}{2}, 0,0,-\frac{1}{2}, 0}+(Q)_{0, \frac{1}{2}, 0,0,-\frac{1}{2}, 0}, \\
& \left(e_{L}^{c}+u_{L}^{c}\right)_{0,0, \frac{1}{2}, 0,0, \frac{1}{2}}+\left(N_{L}^{c}+d_{L}^{c}\right)_{0,0, \frac{1}{2}, 0,0, \frac{1}{2}}+(L)_{0,0, \frac{1}{2}, 0,0,-\frac{1}{2}}+(Q)_{0,0, \frac{1}{2}, 0,0,-\frac{1}{2}} .
\end{aligned}
$$

with

$$
e_{L}^{c} \equiv\left[\left(1, \frac{3}{2}\right) ;(1,1)\right]_{(1,1 / 2,1)} ;
$$

$\dagger$ Note that we could have instead defined the weak hypercharge to be $U(1)_{Y}=\frac{1}{3} U(1)_{C}-\frac{1}{2} U(1)_{L}$. This amounts to the same redefinition of fields between the straight and flipped $S U(5)$. In this paper we will use the definition in Eq. 2.5. 


$$
\begin{aligned}
u_{L}^{c} & \equiv\left[\left(\overline{3},-\frac{1}{2}\right) ;(1,-1)\right]_{(-2 / 3,1 / 2,-2 / 3)} \\
Q & \equiv\left[\left(3, \frac{1}{2}\right) ;(2,0)\right]_{(1 / 6,1 / 2,(2 / 3,-1 / 3))} \\
N_{L}^{c} & \equiv\left[\left(1, \frac{3}{2}\right) ;(1,-1)\right]_{(0,5 / 2,0)} \\
d_{L}^{c} & \equiv\left[\left(\overline{3},-\frac{1}{2}\right) ;(1,1)\right]_{(1 / 3,-3 / 2,1 / 3)} \\
L & \equiv\left[\left(1,-\frac{3}{2}\right) ;(2,0)\right]_{(-1 / 2,-3 / 2,(0,1))}
\end{aligned}
$$

where we have used the notation

$$
\left[\left(S U(3)_{C} \times U(1)_{C}\right) ;\left(S U(2)_{L} \times U(1)_{L}\right)\right]_{\left(Q_{Y}, Q_{Z^{\prime}}, Q_{\mathrm{e} . \mathrm{m} .}\right)}
$$

and for the doublets we have written the electric charge of the two components. In the superstring-derived standard-like models the vectors $b_{1}, b_{2}$ and $b_{3}$ are the only vectors in the additive group $\Xi$ which give rise to spinorial 16 of $S O(10)$. In this case the three light generations are identified unambiguously with the states from these three sectors.

The Neveu-Schwarz sector produces, in addition to the gravity and the gauge boson multiplets, three pairs of electroweak doublets, three pairs of $S O(10)$ singlets which are charged with respect to $U(1)_{1,2,3}$, and three states which are singlets of the entire four dimensional gauge group. In the realistic free fermionic models, typically there is one additional sector which produces electroweak doublet representations. Usually, this sector is a combination of two of the vectors which extend the NAHE set. For example, in the model ref. [12], the combination $b_{1}+b_{2}+\alpha+\beta$ produces one pair of electroweak doublets, one pair of color triplets and five pairs of $S O(10)$ singlets which are charged with respect to the $U(1)$ currents of the observable gauge group. All the states from the NS sector as well as the states from the sector $b_{1}+b_{2}+\alpha+\beta$, which carry Standard Model charges, are obtained by GSO projections from the 10 and $\overline{10}$ representation of $S O(10)$. Obviously, the $S O(10)$ singlet fields from these sector do not carry any Standard Model charges.

The states above complete the representations that we identify with possible representations of the Standard Model. In addition to the Standard Model states, semirealistic superstring models may contain additional multiplets, in the 16 and $\overline{16}$ representation of $S O(10)$, in the vectorial 10 representation of $S O(10)$, or the 27 and $\overline{27}$ of $E_{6}$. Such states can pair up to form super massive states. They can mix with, and decay into, the Standard Model representation unless some additional symmetry, which forbids their decay, is imposed. For example, in the flipped $S U(5)$ superstring models [6], two of the additional vectors which extend the NAHE set produce an additional 16 and $\overline{16}$ representation of $S O(10)$. These states are used in the flipped $S U(5)$ model to break the $S U(5) \times U(1)$ symmetry to $S U(3) \times S U(2) \times U(1)$. 
All the states above fit into standard representation of the grand unified group which may be, for example, $S O(10)$ or $E_{6}$. They carry the standard charges under the Standard Model gauge group or of its GUT extensions. The superstring models, however, contain additional states that cannot fit into multiplets of the original unifying gauge group. In the next section we enumerate the states that appear in free fermionic models. These states have important cosmological implications which we study below.

\section{$3 \quad$ Exotic matter in free fermionic models}

At the level of the NAHE set the observable gauge symmetry is $S O(10) \times S O(6)^{3}$ and the number of spinorial 16 representations of $S O(10)$ is 48 . The basis vectors of the NAHE set are seen to correspond to $Z_{2} \times Z_{2}$ orbifold compactification at an enhanced symmetry point in the toroidal compactification space [26]. To reduce the number of generations, and to break the $S O(10)$ gauge group to the Standard Model gauge symmetry, additional basis vectors are added to the NAHE set. Three additional basis vectors are needed to obtain three generations, one from each of the sectors $b_{1}, b_{2}$ and $b_{3}$. The additional basis vectors which break the $S O(10)$ gauge symmetry correspond to Wilson lines in the orbifold formulation.

Adding to the untwisted sector the three twisted sectors $b_{1}, b_{2}$ and $b_{3}$ results in additional massless states from these sectors. In the same way adding the sectors $\{\alpha, \beta, \gamma\}$ to the NAHE set results in additional massless spectrum from combinations of these basis vectors with those of the NAHE set. However, since these sectors correspond to "Wilson lines" they give rise to massless states that do not fit into representations of the original $S O(10)$ symmetry. As a result the massless spectrum contains states with fractional charges under the unbroken $U(1)$ generators of the original non-Abelian gauge group. This is a new feature of superstring models. Due to the absence of adjoint representations, at least in superstring models with level one gauge groups, "Wilson line" breaking is the only available mechanism to break the unifying gauge symmetry perturbatively. Therefore, the appearance of massless states with fractional $U(1)$ charges is a common phenomenon in superstring models. In many examples the exotic states appear in vector-like representations and can acquire a heavy mass. The "Wilsonian" matter phenomenon is an important feature as it may result in discrete symmetries that may prevent the decay of the exotic massive states into the Standard Model states.

The following exotic matter representations can appear in free fermionic level one models. Sectors that break the $S O(10)$ symmetry to $S O(6) \times S O(4)$ can contain the basis vectors $\alpha$ or $\beta$. Sectors that break the $S O(10)$ symmetry to $S U(5) \times U(1)$ contain the basis vector $\gamma$ with a combination of the other basis vectors. We use the following naming scheme for our particles. An exotic quark state will be denoted by $W_{q_{i}}, W_{\bar{q}_{i}}$ while all the non-colored exotic states will be denoted by $W_{\ell_{i}}, W_{\ell_{i}}$.

From the $S O(6) \times S O(4)$ type sectors we obtain the following exotic states. 
- $\underline{\text { Color triplets: }}$

$$
\begin{aligned}
& {\left[\left(3, \frac{1}{2}\right) ;(1,0)\right]_{(1 / 6,1 / 2,1 / 6)}} \\
& {\left[\left(\overline{3},-\frac{1}{2}\right) ;(1,0)\right]_{(-1 / 6,-1 / 2,-1 / 6)}}
\end{aligned}
$$

Due to its fractional charge under $U(1)_{Y}$ we refer to this state as the sexton. The sexton appears for example in the model of ref. [14 from the sector $1+\alpha+2 \gamma$ and in the model of ref. [7] from the sector $S+b_{2}+b_{4}+\alpha$. The sexton binds with light quarks to form mesons and baryons with fractional electric charges $\pm 1 / 2$ and $\pm 3 / 2$.

- Electroweak doublets:

$$
[(1,0) ;(2,0)]_{(0,0, \pm 1 / 2)}
$$

Such states appear for example in the model of ref. [14 from the sectors $1+b_{i}+$ $\alpha+2 \gamma(i=1,2,3)$. In the model of ref. [11 from the sectors $\xi+b_{1}+\alpha+2 \gamma$ and $\xi+\alpha+2 \gamma$, and in the model of ref. [7] from the sectors $b_{1}+\alpha, b_{1}+b_{2}+b_{4}+\alpha$, $b_{2}+b_{3}+b_{5}+\alpha, b_{4}+\alpha, b_{1}+b_{4}+b_{5}+\alpha$.

We also obtain from this type of sectors fractionally charged $S U(3)_{C} \times S U(2)_{L}$ singlets. In the $S O(6) \times S O(4)$ models these states are doublets of $S U(2)_{R}$ which have zero $U(1)_{C}$ charge and the $S U(3)_{C}$ singlet in the quartets of $S U(4)$ with zero $U(1)_{L}$ charge. In the standard-like models these states are $S U(3)_{C} \times S U(2)_{L}$ singlets with electric charge $Q_{\text {e.m. }}= \pm 1 / 2$.

- Fractionally charged $S U(3)_{C} \times S U(2)_{L}$ singlets :

$$
\begin{aligned}
& {[(1,0) ;(1, \pm 1)]_{( \pm 1 / 2, \mp 1 / 2, \pm 1 / 2)}} \\
& {[(1, \pm 3 / 2) ;(1,0)]_{( \pm 1 / 2, \pm 1 / 2, \pm 1 / 2)}}
\end{aligned}
$$

From sectors which break the $S O(10)$ symmetry into $S U(5) \times U(1)$ we obtain exotic states with fractional electric charge $\pm 1 / 2$

- Fractionally charged $S U(3)_{C} \times S U(2)_{L}$ singlets :

$$
[(1, \pm 3 / 4) ;(1, \pm 1 / 2)]_{( \pm 1 / 2, \pm 1 / 4, \pm 1 / 2)}
$$

In general the fractionally charged states may transform under a non-Abelian hidden gauge group in which case the fractionally charged states may be confined. For example, in the "revamped" flipped $S U(5)$ model [6] the states with fractional charge $\pm 1 / 2$ transform as 4 and $\overline{4}$ of the hidden $S U(4)$ gauge group. In other models these states may be singlets of all the non-Abelian group factors. Such states appear for example in the model of ref. [12] from the sectors $\left\{b_{1}+b_{2}+\alpha+\beta \pm \gamma, b_{1}+b_{3}+\right.$ 
$\left.\alpha+\beta \pm \gamma, b_{2}+b_{3}+\alpha+\beta \pm \gamma, b_{1}+b_{2}+b_{3}+\alpha+\beta \pm \gamma\right\}$. In the model of ref. [11], they appear from the sector $\left\{ \pm \gamma, \xi \pm \gamma, 1+b_{4} \pm \gamma, \xi+1+b_{4} \pm \gamma, \xi+b_{3} \alpha \pm \gamma\right\}$.

Finally in the superstring derived standard-like models we may obtain exotic states from sectors which are combinations of the $S O(6) \times S O(4)$ breaking vectors and $S U(5) \times U(1)$ breaking vectors. These states then carry the standard charges under the Standard Model gauge group but carry fractional charges under the $U(1)_{Z^{\prime}}$ gauge group. The following exotic states are obtained:

- color triplets :

$$
\begin{aligned}
& {\left[\left(3, \frac{1}{4}\right) ;\left(1, \frac{1}{2}\right)\right]_{(-1 / 3,-1 / 4,-1 / 3)}} \\
& {\left[\left(\overline{3},-\frac{1}{4}\right) ;\left(1, \frac{1}{2}\right)\right]_{(1 / 3,1 / 4,1 / 3)}}
\end{aligned}
$$

In ref. [24, due to its potential role in string gauge coupling unification [27, 25], we referred to this state as "the uniton". Such states appear for example in the model of ref. [12] from the sector $b_{2}+b_{3}+\alpha \pm \gamma$, in the model of ref. [14] from the sectors $b_{1,2}+b_{3}+\beta \pm \gamma$, and in the model of ref. [11] from the sectors $\left\{b_{3}+\alpha \pm \gamma, b_{1}+b_{2}+\right.$ $\left.b_{4}+\alpha \pm \gamma\right\}$.

- electroweak doublets :

$$
\left[\left(1, \pm \frac{3}{4}\right) ;\left(2, \pm \frac{1}{2}\right)\right]_{( \pm 1 / 2, \pm 1 / 4,(1,0) ;(0,-1))}
$$

Unlike the previous electroweak doublets, these electroweak doublets carry the regular charges under the standard model gauge group but carry "fractional" charge under the $S O(10)$ symmetry. Electroweak doublets of these type appear for example in the model of ref. [12] from the sector $b_{1}+b_{3}+\alpha \pm \gamma$. In the model of ref. [11] from the sectors $\left\{b_{3}+\alpha \pm \gamma, b_{1}+b_{2}+b_{4}+\alpha \pm \gamma\right\}$.

Finally, in the superstring derived standard-like models we also obtain states which are Standard Model singlets and carry "fractional" charges under the $U(1)_{Z^{\prime}}$ symmetry.

- Standard model singlets with "fractional" $U(1)_{Z^{\prime}}$ charge :

$$
\left[\left(1, \pm \frac{3}{4}\right) ;\left(1, \mp \frac{1}{2}\right)\right]_{(0, \pm 5 / 4,0)}
$$

These states may transform under a non-Abelian hidden gauge group or may be singlets of all the non-Abelian group factors. This type of Standard Model singlet appears in all the known free fermionic standard-like models. For example, 0 in the model of ref. [14 they are obtained from the sectors $b_{1,2}+b_{3}+\beta \pm \gamma$. In the model of ref. [11] they appear from the sectors $\left\{b_{1}+b_{2}+b_{3}+\alpha \pm \gamma, b_{2}+b_{3}+b_{4}+\alpha \pm \gamma, 1+\right.$ $\left.b_{1}+b_{2}+\alpha \pm \gamma, 1+b_{3}+b_{4}+\alpha \pm \gamma, b_{3}+\alpha \pm \gamma, b_{1}+b_{2}+b_{4}+\alpha \pm \beta\right\}$. 
There are several important issues that are important to examine with regard to the exotic states. Since some of these states carry fractional charges, it is desirable to make them sufficiently heavy or sufficiently rare. All the exotic matter states appear in vector-like representations. They can therefore obtain mass terms from renormalizable or higher order terms in the superpotential. We must then study the renormalizable and nonrenormalizable superpotential in the specific models. The cubic level and higher order terms in the superpotential are extracted by applying the rules of ref. 28]. The problem of fractionally charged states was investigated in ref. [22] for the model of ref. [11]. In the notation of ref. [11] the massless states $\left\{V_{41}, V_{42}, V_{43}, V_{44}, V_{47}, V_{48}, V_{49}, V_{50}\right\}$ are of the form of Eq. (3.5); the massless states $\left\{V_{45}, V_{46}, V_{51}, V_{52}\right\}$ are of the form of Eq. (3.3); the massless states $\left\{H_{1}-H_{14}\right\}$ are of the form of Eq. (3.6); the massless colored states $\left\{H_{33}, H_{40}\right\}$ are of the form of Eq. (3.8) and the the massless weak doublets $\left\{H_{34}, H_{41}\right\}$ are of the form of Eq. (3.9). The remaining exotic states, $\left\{H_{15}-H_{32}, H_{35}-H_{39}, H_{42}\right\}$, are Standard Model singlets of the form of Eq. (3.10). The cubic level superpotential of the exotic massless states is given by

$$
\begin{aligned}
W_{2} & =\frac{1}{\sqrt{2}}\left\{H_{1} H_{2} \phi_{4}+H_{3} H_{4} \bar{\phi}_{4}+H_{5} H_{6} \bar{\phi}_{4}+\left(H_{7} H_{8}+H_{9} H_{10}\right) \phi_{4}^{\prime}\right. \\
& +\left(H_{11}+H_{12}\right)\left(H_{13}+H_{14}\right) \bar{\phi}_{4}^{\prime}+V_{41} V_{42} \bar{\phi}_{4}+V_{43} V_{44} \bar{\phi}_{4} \\
& \left.+V_{45} V_{46} \phi_{4}+\left(V_{47} V_{48}+V_{49} V_{50}\right) \bar{\phi}_{4}^{\prime}+V_{51} V_{52} \phi_{4}^{\prime}\right\} \\
& +\left[H_{15} H_{16} \phi_{56}^{\prime}+H_{17} H_{18} \bar{\phi}_{56}+H_{19} H_{20} \bar{\phi}_{56}^{\prime}+H_{21} H_{22} \bar{\phi}_{56}\right. \\
& +\left(V_{11} V_{12}+V_{13} V_{14}\right) \phi_{13}+\left(V_{15}+V_{16}\right)\left(V_{17}+V_{18}\right) \phi_{13}+V_{19} V_{20} \phi_{13} \\
& +V_{21} V_{22} \phi_{12}+V_{23} V_{24} \phi_{12}+\left(V_{25}+V_{26}\right)\left(V_{27}+V_{28}\right) \phi_{12}+V_{29} V_{30} \phi_{12} \\
& \left.+V_{31} V_{32} \bar{\phi}_{23}+V_{33} V_{34} \phi_{23}+H_{29} H_{30} \bar{\phi}_{13}+H_{36} H_{37} \phi_{12}\right] .
\end{aligned}
$$

By examining the fractionally charged states and the trilinear superpotential, it is observed that all the fractionally charged states receive a Planck scale mass by giving a VEV to the neutral singlets $\bar{\phi}_{4}, \bar{\phi}_{4}^{\prime}, \phi_{4}, \phi_{4}^{\prime}$ which imposes the additional $\mathrm{F}$ flatness constraint $\left(\phi_{4} \bar{\phi}_{4}^{\prime}+\bar{\phi}_{4} \phi_{4}^{\prime}\right)=0$. The other exotic states which are Standard Model singlets do not receive mass by this choice of flat direction. Therefore, at this level of the superpotential, the fractionally charged states can decouple from the remaining light spectrum. Similarly, the issue of fractionally charged states in the model of ref. [12] was studied in ref. [29] where it was found that all the fractionally charged states receive large mass from renormalizable or nonrenormalizable terms. Similar results were also found in the case of the Gepner models [21].

The second issue that must be examined with regard to the exotic "Wilsonian" matter is the interactions with the Standard Model states. The fractional charges of the exotic states under the unbroken $U(1)$ generators of the $S O(10)$ gauge group, may result in conserved discrete symmetry which forbid their decay to the lighter Standard Model states. In the following we will investigate this question with regard to particular states that appear in specific models. 


\section{Cosmology of "Wilsonian" matter}

\subsection{Introduction}

The "Wilsonian" matter states obtained from the realistic superstring models are heavy and stable. Their mass density has important cosmological implications, since

if they are too abundant they will overclose the universe. This gives a limit on their present relic density $\rho_{0}$

$$
\rho_{0} \leq \rho_{c}=1.054 h^{2} \times 10^{4} \mathrm{eV} \mathrm{cm}^{-3},
$$

where $h$ is defined by the present value of the Hubble constant $H_{0}$

$$
h=\frac{H_{0}}{100 \mathrm{~km} \mathrm{sec}^{-1} \mathrm{Mpc}^{-1}} .
$$

The relic density of a particle depends on its mass, couplings and on the reheating temperature after inflation. If the relic density is the same as (or at least comparable to) the critical density of the universe, it could account for the existence of dark matter in the universe.

Since the "Wilsonian" matter states are heavy, they are non-relativistic at the time of structure formation and are candidates for cold dark matter. Models consisting of only hot dark matter, such as neutrinos of a few $\mathrm{eV}$ mass, are ruled out phenomenologically, since they fail to explain the large-scale structure curve of the universe. Only models which contain cold dark matter, like the mixed (cold + hot) dark matter model [47] and the cold dark matter with extra radiation model [48], survive, except for models with non-standard cosmology scenarios, such as models with non-zero cosmological constant. The heavy "Wilsonian" matter states are candidates for cold dark matter. The possible existence of heavy stable particles in the realistic superstring models provides further motivation to study this class of models.

Since these "Wilsonian" states cannot decay into normal particles, their number density can only change by annihilation processes. In several examples that we study below, the "Wilsonian" states are interacting strongly and therefore remain in thermal equilibrium until they become non-relativistic. We will consider one exception of a $S U(3) \times S U(2) \times U(1)_{Y}$ singlet which interacts weakly. A given "Wilsonian" matter state decouples from the thermal bath when its annihilation rate falls below the expansion rate of the universe. The annihilation rate of a particle is given by

$$
\Gamma=\left\langle\sigma_{\text {ann }}|v|\right\rangle n_{E Q},
$$

where $n_{E Q}$, the number density at the equilibrium, is given by

$$
n_{E Q}= \begin{cases}g_{\mathrm{eff}}\left(\frac{\zeta(3)}{\pi^{2}}\right) T^{3} & \text { relativistic } \\ g_{\mathrm{eff}}\left(\frac{m T}{2 \pi}\right)^{3 / 2} \exp (-M / T) & \text { non-relativistic }\end{cases}
$$


Here $\zeta(3)=1.20206$ is the Riemann zeta function of 3 , and $g_{\text {eff }}$ is the effective number of degrees of freedom of the particle.

In the expanding universe, the evolution equation of the particle number density is described by the Boltzmann equation

$$
\frac{d n}{d t}+3 H n=-\left\langle\sigma_{\text {ann }}|v|\right\rangle\left(n^{2}-n_{E Q}^{2}\right)
$$

where the Hubble constant in the radiation dominated era is a function of the temperature

$$
H=1.66 \sqrt{g_{*}} \frac{T^{2}}{m_{p l}} .
$$

During the radiation dominated era, time $(t)$ and temperature are related by

$$
t=0.301 g_{*}^{-1 / 2} \frac{m_{p l}}{T^{2}} .
$$

It is standard to define the dimensionless parameters $x=M / T$ and the number density in a comoving volume $Y=n / \mathrm{s}_{e}$, where $\mathrm{s}_{e}$ is the entropy density

$$
\mathrm{s}_{e}=\frac{2 \pi^{2}}{45} g_{* s} M^{3} x^{-3}
$$

Here $g_{*}$ and $g_{* s}$ are the relativistic degrees of freedom and are defined by

$$
\begin{aligned}
g_{*} & =\sum_{i=\text { bosons }} g_{i}\left(\frac{T_{i}}{T}\right)^{4}+\frac{7}{8} \sum_{i=\text { fermions }} g_{i}\left(\frac{T_{i}}{T}\right)^{4}, \\
g_{* s} & =\sum_{i=\text { bosons }} g_{i}\left(\frac{T_{i}}{T}\right)^{3}+\frac{7}{8} \sum_{i=\text { fermions }} g_{i}\left(\frac{T_{i}}{T}\right)^{3},
\end{aligned}
$$

where $g_{i}$ and $T_{i}$ are the degrees of freedom and the temperature of a particle $i$, respectively. The Boltzmann equation, Eq. (4.5), describes the evolution of the number density with respect to temperature in a comoving volume,

$$
\frac{d Y}{d x}=-\lambda x^{-2}\left(Y^{2}-Y_{E Q}^{2}\right)
$$

where

$$
\lambda=\frac{x\left\langle\sigma_{\mathrm{ann}}|v|\right\rangle \mathrm{s}_{e}}{H}
$$

In many cases $\lambda$, in the non-relativistic limit, becomes $x$-independent. Equation (4.10) can be solved if we know the annihilation cross section as a function of the temperature. It is known that the relic density of a particle of mass $M$ is given by 
two different expressions, depending upon the two possible temperature regimes at which decoupling occurs

$$
Y_{0}=\left\{\begin{array}{cc}
0.278\left(\frac{g_{\mathrm{eff}}}{\left.g_{* s\left(T_{d e c}\right.}\right)}\right) & M \ll T_{d e c} \\
\frac{3.79 x_{d e c}}{\sqrt{g_{*}} m_{p l} M\langle\sigma|v|\rangle} & M>T_{d e c}
\end{array}\right.
$$

where we have defined $x_{d e c}=M / T_{d e c}$, and $T_{d e c}$ denotes the decoupling temperature. The two cases $M \ll T_{d e c}$ and $M>T_{d e c}$ describe decoupling in relativistic and nonrelativistic regimes, respectively. If we define $s_{e_{0}}$ to be the entropy density of the present universe, then the relic energy density of a massive decoupled particle is given by

$$
\rho_{0}=\mathrm{s}_{e_{0}} Y_{0} M=2.97 \times 10^{3} Y_{0} M \mathrm{~cm}^{-3},
$$

and we can estimate the $\Omega_{0}$ parameter, which is given by the expression

$$
\Omega_{0} h^{2} \equiv \frac{\rho_{0} h^{2}}{\rho_{c}}=\frac{2970 M Y_{0} \mathrm{~cm}^{-3}}{1.05 \times 10^{4} \mathrm{eV} \mathrm{cm}^{-3}} .
$$

Using the fact that the cosmological data set the bound $0.1<\Omega_{0} h^{2}<1$, Eq. (4.14) gives a bound on the mass of a stable particle

$$
M<\frac{3.5}{Y_{0}} \mathrm{eV}
$$

If a massive particle is weakly interacting (WIMP) at high temperature, it can decouple from thermal equilibrium when it is still relativistic. In this case the upper bound for its mass is very low, since the $Y_{0}$ value is too high. This can be deduced from Eq. (4.12). In the case of an inflationary scenario, however, this bound can be raised. We will elaborate over this possibility in some detail in the following sections.

The class of superstring models which we have discussed in the previous sections allow several kinds of stable "Wilsonian" matter states. In the following we will specifically focus our attention on three cosmologically interesting cases.

\subsection{A stable heavy down-like quark: the uniton}

In this section we investigate the possibility that the dark matter is composed of heavy down-like color triplets of the form of Eq. (3.8). This will be our most detailed quantitative and complex analysis, and the discussion with regard to the other potential dark matter candidates from superstring models will be somewhat more qualitative. 


\subsubsection{Motivation: string gauge coupling unification}

The existence of additional colored vector-like states beyond the spectrum of the Minimal Supersymmetric Standard Model is motivated also from a different consideration. Superstring unification predicts that the gauge and gravitational couplings are unified at the string unification scale which is of the order $M_{\text {string }} \approx g_{\text {string }} 5 \cdot 10^{17} \mathrm{GeV}$ with $g_{\text {string }} \sim 0.8$ 31, 32, 33]. Assuming, naively, that the massless states below the string scale consists solely of the Minimal Supersymmetric Standard Model results in disagreement with the experimentally observed values for $\sin ^{2} \theta_{W}\left(M_{Z}\right)$ and $\alpha_{\text {strong }}\left(M_{Z}\right)$. If one assumes naively that the spectrum between the electroweak scale and the unification scale consists solely of the MSSM state then the gauge couplings are seen to intersect at $\sim 2 \times 10^{16} \mathrm{GeV}$ [34]. This discrepancy is usually referred to as the order of magnitude mismatch between the MSSM and string unification scales.

It would seem that in an extrapolation of the gauge parameters over fifteen orders of magnitude, a problem involving a single order of magnitude would have many possible resolutions. Indeed, in string theory there are many possible effects that can a priori account for the discrepancy. For example, there could be large corrections to the gauge couplings due to the infinite tower of heavy string modes [35]. Alternatively, additional matter [36, 27, 9, 25] or gauge structure beyond the MSSM could reconcile the two scales. Yet another possibility is that the weak hypercharge normalization in string theory is not necessarily the one that is traditionally found in GUTs and could take values that would yield meeting of the couplings at the string scale [37.

Surprisingly, however, the discrepancy is not easily resolved. In ref. [25] the stringscale gauge coupling unification problem was investigated in detail in the context of the realistic free fermionic superstring models. It was shown, in a wide range of realistic free fermionic models, that heavy string threshold corrections, non-standard hypercharge normalizations, light SUSY thresholds or intermediate gauge structure do not resolve the problem. Instead, the problem may only be resolved due to the existence of additional intermediate matter thresholds, beyond the MSSM spectrum. This additional matter takes the form of additional color triplets and electroweak doublets, in vector-like representations. Remarkably, some string models contain in their massless spectrum the additional states with the specific weak hypercharge assignments, needed to achieve string scale unification [27. Perhaps even more intriguing is the fact that in some models the additional states are those that arise due to the "Wilson line" breaking. Thus, the existence of stable "Wilsonian" states at intermediate energy scales is motivated also from requiring consistency of the stringscale gauge coupling unification with the observed low energy gauge parameters.

The mass scale of the additional color triplets is constrained by requiring agreement between the low energy gauge observables and unification of the gauge couplings at the string scale. In ref. 25] the following two constraint equations on the inter- 
mediate matter mass scales were obtained,

$$
\begin{aligned}
10.29 & <\sum_{i}\left(b_{2_{i}}-b_{1_{i}}\right) \ln \frac{M_{S}}{M_{i}}<14.14 \\
18.57 & <\sum_{i}\left(b_{3_{i}}-b_{1_{i}}\right) \ln \frac{M_{S}}{M_{i}}<30.58,
\end{aligned}
$$

where $M_{S}$ is the string unification scale, $M_{i}$ are the mass scales of the intermediate thresholds, and $\left\{b_{3}, b_{2}, b_{1}\right\}_{i}$ are the corresponding one-loop $\beta$-function coefficients of $S U(3)_{C} \times S U(2)_{L} \times U(1)_{Y}$. Eqs. (4.16), (4.17) can now be used to constrain the mass scale of the additional color triplets. A variety of possible combinations exist. For example, in the model of ref. [27] with one pair of light color triplets of the uniton type, $\left\{D_{1}, \bar{D}_{1}\right\}$, we obtain the limits

$$
\text { experimental limit }<M_{3}<1.81 \mathrm{TeV} \text {. }
$$

Setting $M_{3}$ at the upper limit of (4.18), we find

$$
7.2 \times 10^{13} \mathrm{GeV}<M_{2}<2.6 \times 10^{14} \mathrm{GeV},
$$

while for a lower limit of $M_{3} \sim 500 \mathrm{GeV}$ we obtain

$$
3.6 \times 10^{5} \mathrm{GeV}<M_{2}<1.7 \times 10^{6} \mathrm{GeV} .
$$

By contrast, with two triplet pairs of the uniton type, $\left\{D_{1}, \bar{D}_{1}, D_{2}, \bar{D}_{2}\right\}$, degenerate at one mass scale $M_{3}$, we instead find

$$
4.3 \times 10^{6} \mathrm{GeV}<M_{3}<9.5 \times 10^{10} \mathrm{GeV},
$$

so that taking the upper limit for $M_{3}$ yields

$$
7.2 \times 10^{13} \mathrm{GeV}<M_{2}<2.6 \times 10^{14} \mathrm{GeV}
$$

while the lower limit on $M_{3}$ yields

$$
5 \times 10^{12} \mathrm{GeV}<M_{2}<1.8 \times 10^{13} \mathrm{GeV} .
$$

Finally, adding the third pair of color triplets of the sexton type $\left\{D_{3}, \bar{D}_{3}\right\}$, and with all three color triplet pairs degenerate at the scale $M_{3}$, we find

$$
2.4 \times 10^{11} \mathrm{GeV}<M_{3}<7.2 \times 10^{13} \mathrm{GeV}
$$

for which the upper and lower limits respectively yield

$$
\begin{aligned}
& 3.7 \times 10^{14} \mathrm{GeV}<\left(M_{2}\right)_{\text {upper }}<1.1 \times 10^{15} \mathrm{GeV} \\
& 5.7 \times 10^{13} \mathrm{GeV}<\left(M_{2}\right)_{\text {lower }}<2 \times 10^{14} \mathrm{GeV}
\end{aligned}
$$


Clearly, many viable scenarios exist, and the above examples are not exhaustive.

Next we observe that these mass scales can be compatible with the flat directions of the cubic level superpotential. For example, in the model of ref. [27], the relevant cubic level mass terms are

$$
\begin{aligned}
& +\frac{1}{2}\left(\xi_{1} D_{1} \bar{D}_{1}+\xi_{2} D_{2} \bar{D}_{2}\right) \\
& \left.+\frac{1}{\sqrt{2}}\left(D_{1} \bar{D}_{2} \phi_{2}+\bar{D}_{1} D_{2} \bar{\phi}_{1}\right)\right\}
\end{aligned}
$$

and there exist $F$ and $D$ flat vacua in which the $\mathrm{VEV}$ of the singlets in (Eq. 4.26) vanishes. We therefore conclude that indeed there is sufficient freedom in the superstring models that allows the additional color triplets to appear at intermediate mass scales.

\subsubsection{Interactions}

We now discuss the interactions of the uniton with the Standard Model states. In the model of ref. [12] there is one pair of uniton states denoted by $\left\{H_{21}, H_{22}\right\}$ and one pair of exotic doublets denoted by $\left\{H_{15}, H_{16}\right\}$. The following cubic level superpotential terms are obtained

$$
D_{45} H_{18} H_{21}+h_{2} H_{16} H_{17}+h_{45} H_{16} H_{25}+\frac{1}{2}\left(\xi_{1} H_{21} H_{22}+\xi_{2} H_{15} H_{16}\right) .
$$

The states $\left\{H_{18}, H_{17}, H_{25}\right\}$ are Standard Model singlets with "fractional" $U(1)_{Z^{\prime}}$ charge of the form of Eq. (3.10). From Eq. (4.27) we observe that in the model of ref. [12], there are no interactions terms, at order $N=3$, with the states from the sectors $b_{1}, b_{2}$ and $b_{3}$. However, potential interaction terms do appear for the exotic color triplets and electroweak doublets with the color triplets from the sector $b_{1}+b_{2}+\alpha+\beta$ and the electroweak doublets from the Neveu-Schwarz sector. These interaction terms are generated by the breaking of $U(1)_{Z^{\prime}}$. For specific choices of flat directions these terms vanish. However, potential non-vanishing interaction terms may be generated from nonrenormalizable terms. The uniton would be a more appealing dark matter candidate if there existed a gauge symmetry or a local discrete symmetry [39] that forbids the interactions terms to all orders of nonrenormalizable terms.

Next we turn to the model of ref. [14]. The superpotential terms of the exotic color triplets with the Standard Model states were studied in ref. [16]. The potential interaction terms are

$$
\begin{aligned}
& L Q \bar{D}, u_{L}^{c} e_{L}^{c} D, Q Q D, u_{L}^{c} d_{L}^{c} \bar{D}, d_{L}^{c} N_{L}^{c} D \\
& Q D h \\
& \bar{D} \bar{D} u_{L}^{c}
\end{aligned}
$$

For the terms in Eq. (4.28) the type of correlators that have to be checked are of the form $b_{i} b_{j} D \phi^{n}$, where $b_{i}$ and $b_{j}$ represent states from the sectors $b_{i}$ and $b_{j}, D$ 
are the additional color triplets, and $\phi^{n}$ is a string of Standard Model singlets. For the first two pairs of color triplets from the sectors $b_{1,2}+b_{3}+\alpha+\beta$, the operators $b_{i} b_{j} D$ are invariant under the weak hypercharge. However, they break $U(1)_{Z^{\prime}}$ because $Q_{Z^{\prime}}(D)=(1 / 2) Q_{Z^{\prime}}\left(D_{45}\right)$. Thus, $D$ has one half the $U(1)_{Z^{\prime}}$ charge of the triplets from the Neveu-Schwarz and $b_{1}+b_{2}+\alpha+\beta$ sectors. Therefore, all the operators in Eq. (4.28), with $D$ being a triplet from one of the sectors $b_{1,2}+b_{3}+\alpha+\beta$, break $U(1)_{Z^{\prime}}$. Thus, the string $\langle\phi\rangle^{n}$ contains a $U(1)_{Z^{\prime}}$ breaking VEV. However, in this model all the available Standard Model singlets with nontrivial $U(1)_{Z^{\prime}}$ charge transform as 3 and $\overline{3}$ of the Hidden $S U(3)$ gauge group [27]. The $U(1)_{Z^{\prime}}$ charges of the hidden $S U(3)$ triplets are $\pm 5 / 4$. The $U(1)_{Z^{\prime}}$ charges of the color triplets from the exotic "Wilson line" sectors are $\pm 1 / 4$ (see table 1 ). The last pair of color triplets has "fractional" weak hypercharge $Q_{Y}= \pm 1 / 12$. Consequently, terms of the form of Eq. (4.28), with $D$ being a triplet from one of the exotic "Wilson line" sectors, cannot be formed in this model. This result was verified by a computer search of the relevant nonrenormalizable terms up to order $N=12$.

The term in Eq. (4.29) breaks $U(1)_{Z^{\prime}}$. Therefore, the product $\phi^{n}$ in a potential non-vanishing higher order term must break $U(1)_{Z^{\prime}}$. The available fields are $\left\{H_{1}, H_{2}, \bar{H}_{1}, \bar{H}_{2}\right\}$. These fields transform as 3 and $\overline{3}$ of the hidden $S U(3)_{H}$ gauge group with opposite charges under $U(1)_{Z^{\prime}}$ for the states and the bared states. Consequently, to obtain terms which are invariant under the hidden $S U(3)_{H}$ gauge group, some of the $\left\{V_{1,2,3}, \bar{V}_{1,2,3}\right\}$ fields, which transform as 3 and $\overline{3}$ of $S U(3)_{H}$, must get a VEV. Therefore if we impose that the VEVs of the $V_{i}, \bar{V}_{i}$ are suppressed, the terms of the of Eq. (4.29) are suppressed to all orders of nonrenormalizable terms. We remark that there is no phenomenological constraint that requires the VEVs of $V_{i}, \bar{V}_{i}$ to be non-vanishing. In contrast to the model of ref. [12] in which generation mixing is obtained by these VEVs [40, in this model the generation mixing is obtained by the VEVs of the fields $T_{i}, \bar{T}_{i}$. Finally, the term in Eq. (4.30) is invariant under $U(1)_{Y}$ and $U(1)_{Z^{\prime}}$. However the product $\bar{D} \bar{D}$ carries nontrivial charges under $U(1)_{7}$ and $U(1)_{8}$. All the Standard Model singlet fields which are charged under $U(1)_{7}$ and $U(1)_{8}$ transform as $5, \overline{5}$ of $S U(5)_{H}$ or as $3, \overline{3}$ of $S U(3)_{H}$. Consequently, the product $\phi^{n}$ must contain a baryonic factor under $S U(5)_{H}$ or $S U(3)_{H}$. Such terms were not found up to order $N=12$. Imposing that the VEVs of $V_{i}, \bar{V}_{i}$ are suppressed, suppresses the terms of the form of Eq. (4.30), because the $U(1)_{7,8}$ charges of the product $5_{H} \cdots 5_{H}$ cannot be canceled. Thus, with this assumption the term $\bar{D} \bar{D} u_{L}^{c}$ is forbidden to all orders of nonrenormalizable terms. To summarize, if we assume that $S U(3)_{H}$ remains unbroken at the Planck scale, it is seen that all the interaction terms in Eqs. (4.28,4.29,4.30 vanish, to all orders or nonrenormalizable terms.

\subsubsection{Uniton dark matter}

In the previous sections we have seen that superstring models in the free fermionic formulation motivate the existence of vector-like heavy stable $S U(3)_{C}$ triplets, $(3,1)_{1 / 3}$, 
$(3,1)_{1 / 6}$. In this notation the second quantum number denotes their $S U(2)_{L}$ content and the subscript denotes the hypercharge. The uniton [24], i.e. the state $(3,1)_{1 / 3}$, is a $d$-type quark and interacts with the color force. In the mass range we are interested in $\left(10^{3}<M<10^{14} \mathrm{GeV}\right)$, the interaction rate is greater than the expansion rate of the universe, unless the number density of these particles is significantly suppressed. In general it is well known that it is hard to envision a scenario in which a particle carrying unbroken gauge charges decouples in a relativistic regime. Thus, the uniton can be decoupled only when it becomes non-relativistic. In the non-relativistic limit $T / M<1$, the annihilation rate of the uniton is given by

$$
\Gamma \simeq \frac{\pi N \alpha_{s}^{2}}{M^{2}} n_{E Q},
$$

where $M$ is the mass of the uniton and $\alpha_{s} \equiv g_{s}^{2} / 4 \pi$ is the gauge coupling at the decoupling temperature. In this equation $n_{E Q}$ is the number density of the uniton in the non-relativistic limit, while $N$ is obtained by summing over all the available annihilation channels and is given by

$$
N=\sum_{f} c_{f} .
$$

A discussion of this result can be found in appendix B where we present a complete calculation of all the main channels of annihilation of this particle. The amplitudes $c_{f}$ are obtained by taking the non-relativistic limit of the cross sections. The calculation is implemented in the case of $N=1$ supersymmetric QCD with heavy quark-antiquark $(Q \bar{Q})$ initial states and light gluinos $(\tilde{g})$, squarks $(\tilde{q})$, quarks $(q)$ and gluons $(g)$ in the final state $(Q \bar{Q} \rightarrow q \bar{q}, Q \bar{Q} \rightarrow g g, Q \bar{Q} \rightarrow \tilde{q} \tilde{q}, Q \bar{Q} \rightarrow \tilde{g} \tilde{g})$. We refer to figs. 5-10 for details.

We obtain $c_{q}=4 / 3$ for quarks, $c_{g}=14 / 27$ for gluons, $c_{\tilde{q}}=2 / 3$ for squarks and $c_{\tilde{g}}=64 / 27$ for gluinos. Using these results we get the expression for $\lambda$ (see Eq. (4.10)

$$
\lambda=0.83 N \alpha_{s}^{2} \frac{g_{*} s}{\sqrt{g_{*}}} \frac{m_{p l}}{M} .
$$

At this point we can impose the decoupling condition $d Y / d x \simeq 0$ [44] which gives

$$
x_{d e c}=\ln [(2+c) \lambda a c]-\frac{1}{2} \ln \{\ln [(2+c) \lambda a c]\},
$$

where $a=0.145\left(g / g_{* s}\right)$ and $c$ is $Y\left(T_{d e c}\right) / Y_{E Q}\left(T_{d e c}\right)$, the latter being of order one. Therefore we estimate a decoupling temperature of the form

$$
T_{d e c} \simeq \frac{M}{\ln \left(m_{p l} / M\right)} .
$$


Using these results we can estimate the value of the number density in a comoving volume in the present universe

$$
Y_{0}=\frac{3.79 x_{d e c}}{\sqrt{g_{*}} m_{p l} M\langle\sigma|v|\rangle}=3.8 \frac{M \ln \left(m_{p l} / M\right)}{N \alpha_{s}^{2} \sqrt{g_{*}} m_{p l}} .
$$

We have set $g_{*}=g_{* s}$, since the decoupling temperature is high 44].

Using this condition and Eq. (4.15), we get an upper bound on the mass of the uniton

$$
M<10^{5} \alpha_{s}\left(N \sqrt{g_{*}} \ln \left(m_{p l} / M\right)\right)^{1 / 2} \mathrm{GeV} .
$$

We now turn to discuss the case in which we have inflation. The effect of inflation is important if the decoupling temperature is greater than the reheating temperature $\left(T_{\text {dec }} \gg T_{R}\right)$. If $T_{R} \ll T_{\text {dec }}$ the uniton will be diluted away, although it can be regenerated after reheating by out-of-equilibrium production 44. Therefore we can approximately set the initial density of the uniton to be zero and obtain the relation

$$
\frac{d Y}{d x}=\lambda x^{-2} Y_{E Q}^{2}
$$

with $Y_{E Q}=0.145 g_{e f f} / g_{*} x^{3 / 2} e^{-x}$. Integrating this equation from the reheating temperature down to the present temperature we get

$$
Y_{0}=\frac{\lambda g_{\text {eff }}^{2}}{2}\left(\frac{0.145}{g_{*}}\right)^{2}\left(x_{r}+\frac{1}{2}\right) e^{-2 x_{r}},
$$

where $x_{r} \equiv M / T_{R}$ and

$$
\Omega_{0} h^{2} \simeq 9 \times 10^{3} N \alpha_{s}^{2} g_{e f f}^{2} \frac{m_{p l}}{\mathrm{eV}}\left(\frac{200}{g_{*}}\right)^{1.5}\left(x_{r}+\frac{1}{2}\right) e^{-2 x_{r}},
$$

from which we derive a bound on the mass of the uniton

$$
M>T_{R}\left[25+\frac{1}{2} \ln \left(\frac{M}{T_{R}}\right)\right] .
$$

Without inflation, we have a strict bound on the mass of the uniton which is around $10^{5} \mathrm{GeV}$. However, inflation can raise the mass bound to any arbitrary order, depending upon the estimated value of the reheating temperature.

In ref. [50] it was pointed out that an exotic stable heavy quark, which decouples at high temperature, can annihilate after color confinement. At low temperature heavy quarks will form bound states of finite size $a_{f}$ with ordinary quarks. Since the temperature at confinement is quite small compared to the heavy quark mass, the scattering cross section of two of these heavy hadrons will approximately be equal to their geometric cross section $\sigma \sim 4 \pi a_{f}^{2}$, with $a_{f}$ about $1 \mathrm{fm}$. We cannot estimate the corresponding annihilation cross section at this temperature. However, it is not 
unreasonable to assume that the two cross sections (annihilation and scattering) are proportional to each other:

$$
\sigma_{\mathrm{ann}}=\eta 4 \pi a_{f}^{2} .
$$

Within this assumption, from the Boltzmann equation we can calculate the value of the relic density

$$
\begin{aligned}
\frac{d Y}{d x} & =-\frac{\langle\sigma|v|\rangle s}{x H} Y^{2} \\
& =-M m_{p l} \eta \pi a_{f}^{2} x^{-2.5} Y^{2} .
\end{aligned}
$$

Notice that we have set $Y_{E Q}$ to be zero since the temperature is much lower than the mass of the uniton.

After integration from confinement temperature to the present temperature, we get

$$
\frac{1}{Y_{f}}-\frac{1}{Y_{i}}=\frac{2}{3} M m_{p l} \eta \pi a_{f}^{2}\left[\left(\frac{T_{i}}{M}\right)^{3 / 2}-\left(\frac{T_{f}}{M}\right)^{3 / 2}\right]
$$

with $i$ and $f$ characterizing the beginning and end of the annihilation period, respectively.

If we assume that this mechanism can give a large suppression to the relic density of the uniton, then we can ignore $T_{f} / M$ and $1 / Y_{i}$ in Eq. (4.44) and the new relic density becomes

$$
Y_{0} \simeq \frac{3 \sqrt{M}}{2 \pi \eta a_{f}^{2} m_{p l} T_{i}^{3 / 2}} .
$$

At this point we need an estimate of $\eta$. If we assume that the scattering and the annihilation cross sections are of same order, i.e. $\eta \simeq 1$, and if we insert the value $T_{i} \sim 1 \mathrm{GeV}$, which is the temperature at the confining phase, the upper bound on the uniton mass can be raised up to $10^{7}-10^{8} \mathrm{GeV}$. However, we do not have any definitive argument which can help us estimate the value of $\eta$. Therefore this issue remains open.

If we ignore this re-equilibrium process, then the mass of the uniton should be smaller than $10^{5} \mathrm{GeV}$ or greater than $\mathcal{O}(10) \times$ reheating temperature, which is the result presented in Eqs. (4.37) and (4.41).

On the other hand, the uniton can make a stable neutral bound state and as such can be component of dark matter. This issue is crucially connected to the question of whether the neutral $\left(U_{0}\right)$ or the the charged $\left(U_{-1}\right)$ bound state is the lightest between the two possible states of this meson. We refer to appendix A for a discussion of this point. There we use arguments based both on QCD potential models and results from the heavy quark effective theory to conclude that the possibility of having $U_{0}$ as the lowest state is not ruled out. The splitting between the two states is likely to be of $1-2 \mathrm{MeV}$, which is of the order of the electromagnetic mass splitting, and allows a conversion of $U_{-1}$ into $U_{0}$ by beta decay. 
If $U_{0}$ is a component of dark matter then its relic energy density should have the same order of magnitude as the critical energy density $\rho_{c}$. Therefore from Eq. (4.14) we can estimate the mass of the $U_{0}$ both in the case of inflation and without. In the first case we get

$$
M \simeq 10^{5} \alpha_{s}\left(N \sqrt{g_{*}} \ln \left(m_{p l} / M\right)\right)^{1 / 2} \mathrm{GeV},
$$

while in the latter case the estimated mass is given by

$$
M \simeq T_{R}\left[25+\frac{1}{2} \ln \left(\frac{M}{T_{R}}\right)\right] .
$$

We remark that there are three windows (in the parameter space $M / \sigma_{p}$, with $\sigma_{p}$ denoting the scattering cross section on protons) for strongly interacting dark matter (such as $U_{0}$ ) [49] which possibly meet our requirements. The first window is in the relatively low mass range $\left(10 \mathrm{GeV}<M<10^{4} \mathrm{GeV}\right)$ and in the range $10^{-24}<\sigma_{p}<10^{-20} \mathrm{~cm}^{2}$. In other two windows it is required to have $10^{5} \mathrm{GeV}$ $<M<10^{7} \mathrm{GeV}$ and $M>10^{10} \mathrm{GeV}$, respectively, assuming a cross section, in both cases, less than $10^{-25} \mathrm{~cm}^{2}$. These constraints include bounds from various experiments (such as experiments performed using solid state cosmic-ray detectors and plastic track cosmic-ray detectors) and from cosmological consideration (such as the galactic halo infall rate and the life-time of neutron stars [45]).

\subsection{Fractionally charged matter}

As we have discussed in the previous sections, among the "Wilsonian" states predicted by the free fermionic superstring models in the low energy limit, there are also heavy particles which are $S U(2)_{L}$ doublets and singlets (and which are $S U(3)$ singlets), $(1,2)_{0},(1,1)_{1 / 2}$. These two states are lepton-like, are stable and have fractional electric charge $\pm 1 / 2$. The behavior of these two states at high temperature is supposed to be the same as in the uniton case since the three gauge couplings become of the same order.

We have also seen that superstring models contain fractionally charged vectorlike quarks $(3,1)_{1 / 6}$. We refer to this particle as the sexton. This particle is expected to form bound states with $u, d$ quarks (since it is a color triplet) and can make stable baryons and mesons with fractional electric charge of $\pm 1 / 2$ and $\pm 3 / 2$. The experimental searches for free quarks in various materials show that the upper bound on the number density of fractionally charged particle should be smaller than $10^{-19} \sim$ $10^{-26}$ [51]. This implies that the relic density of fractionally charged matter can be estimated to be

$$
Y_{0}^{F C}<10^{-19} Y_{0}^{B}<10^{-19} \frac{\mathrm{eV}}{m_{p}} \sim 10^{-28} .
$$

This result almost excludes the possibility of fractionally charged dark matter since from Eq. (4.15), the lower bound of the masses of fractionally charged states should 
be given by

$$
M \sim \frac{\mathrm{eV}}{Y_{0}^{F C}}>10^{19} \mathrm{GeV} .
$$

Since the sexton is a color triplet, we can estimate its relic density $Y_{0}$ by the same way as in the uniton case (see (4.36) and (4.39). It is clear that the relic density of the sexton cannot satisfy the constraint given in Eq. (4.48) without inflation if the mass of the sexton is greater than $1 \mathrm{eV}$. Instead, if the mass is lighter than 10 $\mathrm{eV}$, then the sexton behaves like a light quark $(u, d)$ and is confined in a hadron bound state. However, the densities of fractionally charged hadron bound state at low temperature are severely constrained [51]. Therefore, string models which predict light stable fractionally charged particles cannot survive without inflation, since all these fractionally charged particles should be diluted away. Another possible scenario is that the states which carry fractional charges also transform under a non-Abelian gauge group in the hidden sector in which case the fractionally charged states are confined [6]. In this case the fractionally charged states form bound states which are integrally charged [20]. It should be noted, however, that in this scenario one must insure that the neutron star constraints [45] are not violated.

From Eq. (4.39) we can also derive a bound on the relic density of these particles in an inflationary scenario,

$$
Y_{0}=\frac{\lambda g_{\text {eff }}^{2}}{2}\left(\frac{0.145}{g_{*}}\right)^{2}\left(x_{r}+\frac{1}{2}\right) e^{-2 x_{r}}<10^{-28} .
$$

In this equation $\lambda$ is approximately the same as for the uniton case $\sim N \alpha^{2} \sqrt{g_{*}} m_{p l} / M$. Therefore we have the approximate bound,

$$
\frac{M}{T_{R}}>38+\log \left(\frac{T_{R}}{10^{9} \mathrm{GeV}}\right)
$$

In order to evade the experimental constraints on the relic densities of fractionally charged bound states, it is mandatory to show that the density of these states is suppressed. Our arguments on this matter are quite different from those presented in the earlier literature [50]. Two new elements appear in our analysis: $a$ ) the value of the charge of the sexton $( \pm 1 / 6)$, which is different from the usual quark charge assignment $( \pm 1 / 3, \pm 2 / 3)$; and $b)$ the presence of fractionally charged leptons.

At the confinement temperature, the sexton (denoted $\sigma$ ) can form neutral and charged color singlet bound states $(\sigma \sigma \sigma, q \sigma \sigma, q q \sigma, \bar{q} \sigma)$ and their corresponding antiparticle $(\bar{\sigma} \bar{\sigma} \bar{\sigma}, \bar{q} \bar{\sigma} \bar{\sigma}, \bar{q} \bar{q} \bar{\sigma}, q \bar{\sigma})$. It is not hard to show that the number of fractionally charged bound states which can be generated in hadronic reactions is not small. For instance we can classify all the possible conversion processes for both integer and fractional final states. Typical examples of these processes are

$$
q q \sigma+q q \sigma \text { (frac.) } \rightarrow q \sigma \sigma+q q q \text { (int.) }
$$




$$
\begin{aligned}
& \bar{q} \sigma+q q \sigma \text { (frac.) } \rightarrow q \sigma \sigma+\bar{q} q \text { (int.) } \\
& \bar{q} \sigma+q \bar{\sigma} \text { (frac.) } \rightarrow \sigma \bar{\sigma}+q \bar{q} \text { (int.) } \\
& q q \sigma+q \bar{\sigma} \text { (frac.) } \rightarrow \sigma \bar{\sigma}+q q q \text { (int.) }
\end{aligned}
$$

and their converses. Integer charged final states can be easily reconverted into fractionally charged states due to the large amount of ordinary particles $(q \bar{q}$ and $q q q)$ present in the thermal bath. As we can see from (4.52), annihilation channels $(\sigma \bar{\sigma})$ are always present, which in turn can reduce the total number of sexton bound states. This number has been estimated in the case of the uniton in the previous sections. As the temperature goes down due to the expansion of the universe, it is possible that the remaining fractional hadrons will form bound states of integer charge with fractionally charged heavy leptons $(1,2)_{0}$ and $(1,1)_{1 / 2}$ (in our conventions $\left.Q_{\text {e.m. }}=T_{3}+Y\right)$, therefore making neutral heavy hydrogen-type bound states (e.g. $\left.B_{1 / 2}+L_{-1 / 2} \rightarrow H_{0}\right)$. We can estimate the cross section for this process as follows.

At a temperature of few $\mathrm{eV}$, the fractionally charged bound state $B_{1 / 2}$ or $L_{-1 / 2}$ can capture an electron and form a bound state of radius $a_{B}$ (Bohr radius) which is hydrogen-like fractionally charged bound state $\left(B_{1 / 2}+e^{-}\right)$. Therefore we can assume that the cross section for two of these fractionally charged bound state to interact and form a neutral bound state is proportional to the scattering cross section of two hydrogen-like atoms

$$
\sigma=\eta 4 \pi a_{B}^{2}
$$

Then we can calculate the new relic density from the Boltzmann Eq. (4.10), assuming that $Y_{E Q}$ is very small due to the exponential suppression of their number density

$$
\frac{d Y}{d x}=-M m_{p l} \eta \pi a_{B}^{2} x^{-2.5} Y^{2}
$$

Notice that this the same as Eq. (4.43), except that $a_{f}$ is now replaced by $a_{B}$. Therefore we obtain

$$
\frac{1}{Y_{f}}-\frac{1}{Y_{i}}=\frac{2}{3} M m_{p l} \eta \pi a_{B}^{2}\left[\left(\frac{T_{i}}{M}\right)^{3 / 2}-\left(\frac{T_{f}}{M}\right)^{3 / 2}\right] .
$$

This result is the same as in Eq. (4.44), but with a lower temperature value $T_{i}$ and a larger geometrical size $\left(a_{B}>a_{f}\right)$. $Y_{f}$ here denotes the final density of fractionally charged particles whose value is strongly restricted by experimental data [51]. If this neutral bound state $H_{0}$ is a dark matter candidate, it should have the maximally allowed value of $Y_{i}$, i.e. the critical density is

$$
Y_{i} \simeq \frac{3.5 \mathrm{eV}}{M}
$$


and if we insert this value into (4.55) we obtain the conversion rate of fractionally charged bound states to the neutral ones

$$
\frac{Y_{i}}{Y_{f}}-1 \sim \eta\left(\frac{10^{6} \mathrm{GeV}}{M} \frac{T_{i}}{10 \mathrm{eV}}\right)^{1.5}
$$

If we assume, in the most optimistic scenario, that $\eta \approx 1$, from Eqs. (4.48) and (4.57) we easily deduce that a large $Y_{i} / Y_{f}$ ratio is allowed only if the mass $M$ of this bound state is quite small $\left(10^{-20} \mathrm{GeV}\right)$. This is clearly unrealistic since our calculation is valid only if these particles are non-relativistic. Therefore we conclude that light fractionally charged particles cannot be dark matter candidates.

From Eq. (4.55) more generally, we can deduce the relic density of the fractionally charged particles in the form

$$
Y_{f} \simeq \frac{3 \sqrt{M}}{2 \pi \eta a_{B}^{2} m_{p l} T_{i}^{3 / 2}} .
$$

Here, we ignore $1 / Y_{i}$ compared with $1 / Y_{f}$, assuming that this scattering process can suppress the relic density. If we insert the values $a_{B} \simeq 2.7 \times 10^{5} \mathrm{GeV}^{-1}$ and $T_{i} \simeq 10$ $\mathrm{eV}$, it cannot satisfy the constraint (4.48) unless its mass is much smaller than the temperature $T_{i} \simeq 10 \mathrm{eV}$. Since a light particle with non-zero charge has not been detected in the laboratory experiments [51], it is hard to imagine that there is a strong suppression on the relic density of the fractionally charged matter at low temperature. These arguments seem to indicate that string models which contain light fractionally charged states always need inflation. Finally, we comment that fractionally charged states with Planck scale mass could perhaps constitute the dark matter, but in this case the tools that we have used for the analysis may not be adequate.

\subsection{A singlet of $S U(3)_{c} \times S U(2)_{L} \times U(1)_{Y}$}

Another "Wilsonian" state which is obtained in the superstring models, and which is a possible candidate for dark matter is a singlet of $S U(3)_{c} \times S U(2)_{L} \times U(1)_{Y}$, carrying an additional non-standard $U(1)_{Z^{\prime}}$ charge. This type of "Wilsonian" matter state arises due to the breaking of the $S O(10)$ symmetry to $S U(3) \times S U(2) \times U(1)^{2}$ and appears generically in the superstring derived standard-like models. For example, in the model of refs. [27, 14] such states appear from the sectors $b_{1,2}+b_{3}+\beta \pm \gamma$. In this model these states transform as 3 and $\overline{3}$ of a hidden $S U(3)_{H}$ gauge group. As in section 4.2.2 their interactions with the Standard Model states vanish to all orders of nonrenormalizable terms if the $S U(3)_{H}$ is left unbroken.

The $U(1)_{Z^{\prime}}$ symmetry should be broken somewhere in between the weak scale and the Planck scale. In this section we refer to this state as the "W-singlet" $\left(W_{s}\right)$.

After symmetry breaking the $\mathrm{W}$-singlet interaction is suppressed by $1 / M_{Z^{\prime}}^{2}$ and can be classified as a weakly interacting massive particle (WIMP). 


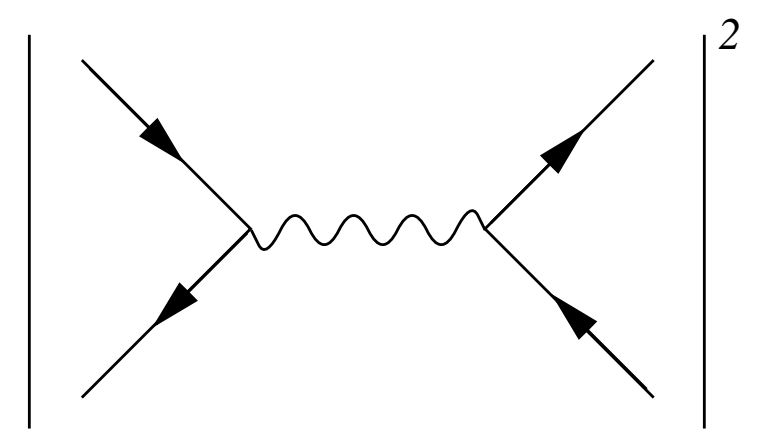

Figure 1: $W_{s} \bar{W}_{s} \longrightarrow f \bar{f}$ decay

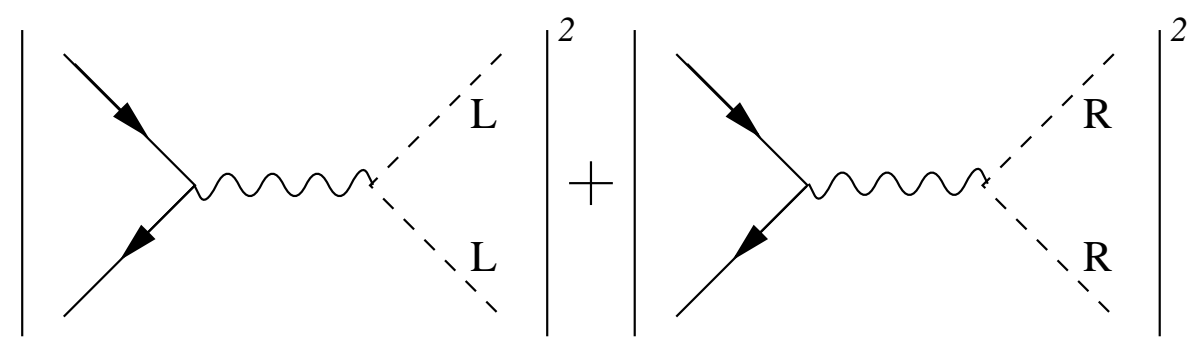

Figure $2: W_{s} \bar{W}_{s} \longrightarrow \tilde{f} \tilde{f}^{*}$ decay

The W-singlet can annihilate into two light Standard Model fermions and into their superpartners. The latter can decay afterwards into two $Z^{\prime}$ gauge bosons. This decay will be suppressed if the gauge boson mass is greater than the mass of $\mathrm{W}$-singlet and this singlet, therefore, will be a stable particle.

Let $M$ be the $\mathrm{W}$-singlet mass, and $M_{Z^{\prime}}$ the mass of the $Z^{\prime}$ gauge boson. Let us consider the total cross section for the annihilation of two W-singlet into two normal fermions and their superpartners.

The two diagrams in figs. 1 and 2 are similar to those shown in figs. 9 and 10 , except for the color factors and for the mass of the s-channel gauge boson, which is now assumed to have a mass $M_{Z^{\prime}}$. We get

$$
\sigma=\frac{4 \pi N_{Z^{\prime}} \sqrt{s}\left(s+2 M^{2}\right)}{3\left(M_{Z^{\prime}}^{2}-s\right)^{2} \sqrt{s-4 M^{2}}},
$$

where $s$ is the square of the C.M. energy $s=4 E_{C M}^{2}$ of each incoming particle and

$$
N_{Z^{\prime}}=\frac{Q_{w}^{2}}{(4 \pi)^{2}} \sum_{f} Q_{f}^{2} .
$$

In this equation $Q_{i}$ are the $U(1)_{Z^{\prime}}$ charges of the $\mathrm{W}$-singlet and of the Standard Model particle $f$. Notice that we have to introduce a $1 / 2$ suppression factor for each scalar partner if there is no mixing between the left-handed sfermion and the right-handed sfermion. 


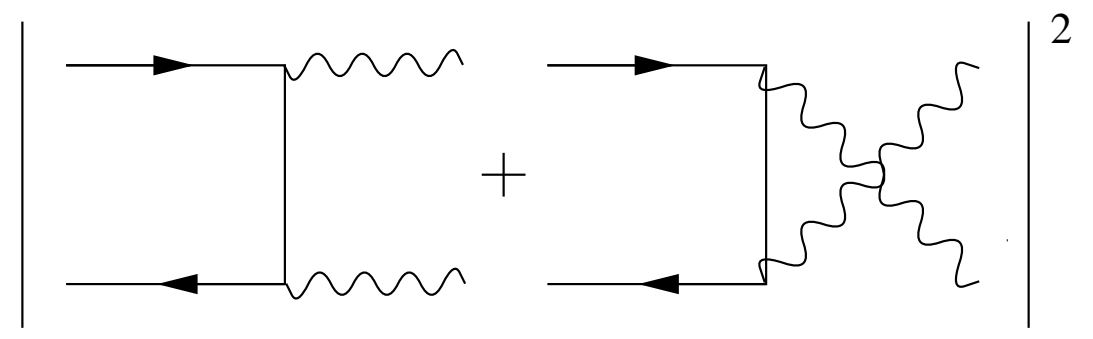

Figure 3: $W_{s} \bar{W}_{s} \longrightarrow Z^{\prime} Z^{\prime}$ decay

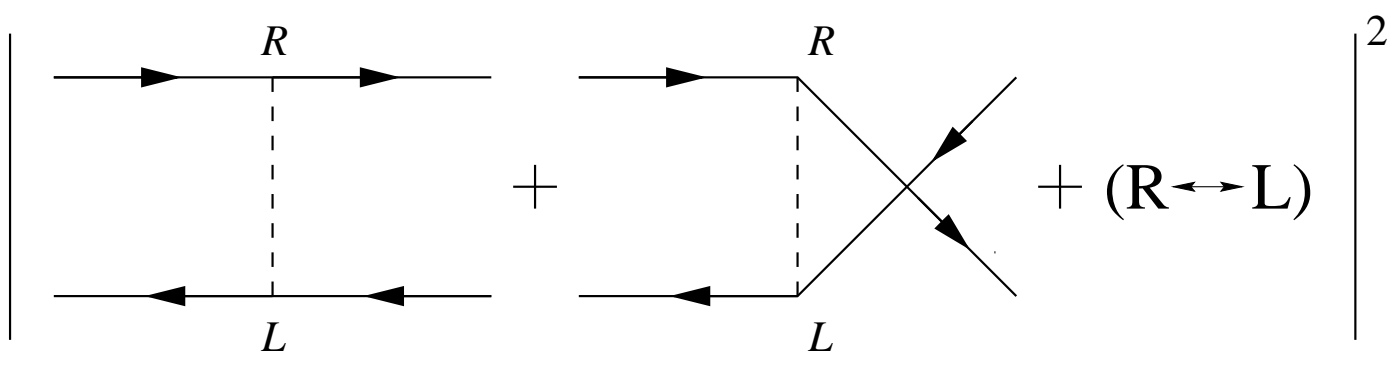

Figure 4: $W_{s} \bar{W}_{s} \longrightarrow \tilde{Z}^{\prime} \tilde{Z}^{\prime}$ decay

Let us comment on the calculation of this cross section, which can be obtained with a slight modifications of the results of Appendix B. For this purpose we start distinguishing two cases: that of a heavy $Z^{\prime}$ and that of a light $Z^{\prime}$. In the case of a heavy $Z^{\prime}$ the only relevant diagrams are those of figs. 1 and 2 . Notice that we do not allow, in this case, $Z^{\prime}$ 's in the final state. Notice also that the annihilation of $W_{s}$ into regular quarks and leptons (and into their supersymmetric partners) is allowed, since all the quarks carry an extra $U(1)_{Z^{\prime}}$ charge. In the case of a leptophobic $Z^{\prime}$ charge [52, 53] we would include in the final states only color states.

In the second case, when $Z^{\prime}$ is light, we include also the channel $W_{s} W_{s} \rightarrow 2 Z^{\prime}$ beside those considered before. The cross section for this last channel is shown in fig. 3 and 4.

The annihilation cross section into two $Z^{\prime}$ is given by

$$
\sigma=\frac{4 \pi \alpha_{w}^{2}}{s A^{2}}\left[\left(s+4 M^{2}\right) A-\left(s^{2}+4 M^{2} s-8 M^{4}\right) \ln \left(\frac{s+A}{s-A}\right)\right],
$$

and the one into two gauginos

$$
\sigma=\frac{4 \pi \alpha_{w}^{2}}{A^{2}}\left[A+2 M^{2} \ln \left(\frac{s+A}{s-A}\right)\right]
$$

where $A=\sqrt{s\left(s-4 M^{2}\right)}$.

Since the decoupling temperature of WIMP's depends on both the mass and coupling constant, we cannot use the same estimate as in the uniton case.

Here we consider four different cases. 
1) W-singlet mass greater than $M_{Z^{\prime}}$ without inflation.

In this case, the $\mathrm{W}$-singlet is a strongly interacting particle when it decouples from the heat bath and therefore the relic density estimate is the same as in the uniton case. The $W_{s}$ is non-relativistic at decoupling and its total annihilation cross section into two $Z^{\prime}$ s and into two gauginos is given by

$$
\sigma|v|=(1+2) \frac{2 \pi}{M^{2}} \alpha_{w}^{2},
$$

while the corresponding two-fermion/sfermion cross section is

$$
\sigma|v|=\frac{\pi}{M^{2}} N_{Z^{\prime}}
$$

where $\alpha_{w}=Q_{w}^{2} / 4 \pi$.

Although the $W_{s}$ 's are strongly interacting particles at decoupling from the thermal bath, they interact weakly in the present universe since the interaction of this particle with the Standard Model particles goes only through the $U(1)_{Z^{\prime}}$ gauge bosons. This interaction, at the present epoch, is suppressed by the heavy $Z^{\prime}$ gauge boson mass since the symmetry is broken. Therefore the bounds on the three windows for the strongly interacting dark matter which we discussed above (section 4.2) are not valid in this case. This leads to constraints which are similar to those of the uniton

$$
M<10^{5}\left(\left(N_{z}+2 \alpha_{w}^{2}\right) \sqrt{g_{*}} \ln \left(m_{p l} / M\right)\right)^{1 / 2} \mathrm{GeV}
$$

2) W-singlet mass greater than $M_{Z^{\prime}}$ with inflation.

If the reheating temperature is greater than $M_{Z^{\prime}}$, the estimated cross section is the same as for the first case. Using Eq. (4.39) similarly we can estimate the relic density of the $W_{s}$ in an inflationary scenario and obtain a bound on its mass

$$
M>T_{R}\left[25+\frac{1}{2} \ln \left(\frac{M}{T_{R}}\right)\right]
$$

However, if the reheating temperature is less than $M_{Z^{\prime}}$, the regeneration of $W_{s}$ goes through a weak $U(1)_{Z^{\prime}}$ process and the bound given above is not valid. This scenario will be discussed in the fourth case below.

3) W-singlet mass is less than $M_{Z^{\prime}}$ and no inflation.

In this case the $W_{s}$ is a WIMP. Therefore we will assume that decoupling will occur when it is still relativistic. Their number density in the comoving volume is then estimated to be

$$
Y_{0} \equiv \frac{n_{E Q}}{\mathrm{~s}}=0.278 \frac{g_{\mathrm{eff}}}{g_{* s\left(T_{\mathrm{dec}}\right)}} \simeq 1.2 \times 10^{-3}
$$


In Eq. (4.67) we assumed that the particle content is that of the MSSM and that the decoupling temperature is larger than $1 \mathrm{TeV}$. This is a conservative assumption as it will minimize the value of $Y_{0}$ and hence maximize the mass bound. Using this value of $Y_{0}$ in Eq. (4.14) we can derive the upper mass bound

$$
M<3 \mathrm{keV} .
$$

The $W_{s}$ with a few $\mathrm{keV}$ can be a candidate of warm dark matter.

4) W-singlet mass less than $M_{Z^{\prime}}$ with inflation.

A heavy mass WIMP can be a dark matter candidate only in the presence of inflation. In fact, inflation will dilute away all the existing $\mathrm{W}$-singlets which will then be regenerated after reheating. In this limit, i.e. $M<M_{Z^{\prime}}$ and $T_{R}<M_{Z^{\prime}}$, we approximate the $Z^{\prime}$-mediated interaction by a four-point Fermi interaction. In this temperature regime, a $W_{s} \bar{W}_{s}$ pair cannot annihilate into two $Z^{\prime}$ s because it is too massive. We also observe that after supersymmetry breaking the mass of the superpartners of the $Z^{\prime}$ s is of the same order (modulo soft breaking corrections) of the $Z^{\prime}$ mass. Therefore the decay channel of two $W_{s}^{\prime}$ 's into two $Z^{\prime}$ gauginos is forbidden as well.

Thus, we have two limits for the cross section

$$
\sigma|v| \simeq 16 N_{Z^{\prime}} \pi \frac{M^{2}}{M_{Z^{\prime}}^{4}}, \quad \text { if } T_{R}<M
$$

and

$$
\sigma|v| \simeq \frac{8}{3} N_{Z^{\prime}} \pi \frac{s}{M_{Z^{\prime}}^{4}}, \quad \text { if } T_{R}>M
$$

In the non-relativistic case $\left(T_{R}<M\right)$ we can use Eq. (4.39) and obtain

$$
M>T_{R}\left[25+\frac{1}{2} \ln \left(\frac{M^{5}}{M_{Z^{\prime}}^{4} T_{R}}\right)\right] .
$$

We now consider the relativistic case $\left(T_{R}>M\right)$. In this case the value we obtain for $\lambda$ in Eq. (4.38) is temperature dependent. In order to estimate Eq. (4.69) we use the thermal average of the energy squared

$$
\langle s\rangle=4\left\langle E^{2}\right\rangle \simeq\left[\frac{5}{4}\right] 40 T^{2} .
$$

Here the factor [5/4] is only appropriate for fermions. Using this result, it is straightforward to rewrite the Boltzmann equation in the form

$$
\begin{aligned}
\frac{d Y}{d x} & =-\frac{\langle\sigma|v|\rangle_{\mathrm{s}}}{x H} Y_{E Q}^{2} \\
& =-4.3 N_{Z^{\prime}} g_{\mathrm{eff}}^{2} g_{*}^{-1.5} \frac{m_{p l} M^{3}}{M_{Z^{\prime}}^{4}} x^{-4}
\end{aligned}
$$


and get the relic density of this particle by integrating this equation from the reheating temperature to the present temperature:

$$
Y_{0}=1.44 N_{Z^{\prime}} g_{\mathrm{eff}}^{2} g_{*}^{-1.5} \frac{m_{p l} T_{R}^{3}}{M_{Z^{\prime}}^{4}}
$$

If we insert this result into Eq. (4.15) we obtain

$$
\frac{M T_{R}^{3}}{M_{Z^{\prime}}^{4}}<6.9 \times 10^{-25}\left(\frac{10^{19} \mathrm{GeV}}{m_{p l}}\right)\left(\frac{g_{*}}{200}\right)^{1.5} \frac{1}{N_{Z^{\prime}} g_{\mathrm{eff}}^{2}} .
$$

Since there are three unknown parameters $\left(M, M_{Z^{\prime}}, T_{R}\right)$ in Eq. (4.74) we cannot infer a definite value for the mass of the $W_{s}$ and $Z^{\prime}$, but we nevertheless deduce that $Z^{\prime}$ should be very heavy.

There is still another possibility to be considered. The W-singlet can be a triplet of a hidden gauge group. If this gauge group is not broken, then the $\mathrm{W}$-singlets can annihilate into two hidden gauge bosons. This will lead to the same result as for the triplet case (the uniton) and we can obtain similar mass bounds.

\section{Conclusion and discussion}

In this paper we studied the cosmological constraints on the exotic matter states that appear in the massless spectrum of realistic free fermionic superstring models. The free fermionic superstring models are among the most realistic string models constructed to date, and reproduce many of the observed properties of the Standard Model. Among those, the replication of three and only three families and the qualitative spectrum of fermion masses [23, 40]. The realistic nature of the free fermionic models is perhaps not accidental but may reflect deeper properties of string compactification, which are at present unknown. Indeed, the free fermionic models are constructed at a highly symmetric point in the moduli space and the appearance of three generations is deeply rooted in the underlying $Z_{2} \times Z_{2}$ orbifold structure [26].

In the derivation of the Standard Model from superstring theory we start with some larger symmetry which is subsequently broken to the Standard Model. Absence of adjoint representations in the massless spectrum of level one Kac-Moody algebras restricts the possible gauge groups in the effective low energy field theory. Furthermore, proton lifetime constraints motivate the hypothesis that the Standard Model must be derived directly from string theory, without an intermediate nonAbelian gauge symmetry. Within the free fermionic construction the breaking is achieved by constructing boundary condition basis vectors which are equivalent to Wilson lines in the geometrical formulation. The use of Wilson lines to break the non-Abelian gauge symmetries is quite generic in superstring theory.

The breaking of the non-Abelian gauge symmetries by Wilson lines has an important feature: it produces matter representations that do not fit into multiplets of the 
original unbroken gauge symmetry. This is an important feature as it may result in local discrete symmetries that forbid the decay of the "Wilsonian" matter states into the lighter Standard Model particles. Superstring models thus provide an intrinsic mechanism that produces heavy stable states.

The "Wilsonian" matter states are classified by the patterns of symmetry breaking, induced by the "Wilson" lines. In the free fermionic models the underlying $S O(10)$ gauge symmetry is broken to $S O(6) \times S O(4), S U(5) \times U(1)$ or $S U(3) \times S U(2) \times$ $U(1)^{2}$. All three cases give rise to fractionally charged states with $Q_{\text {e.m. }}$. These states may all be confined, they may all be superheavy, or they may diluted by inflation. Nevertheless, it may be worthwhile to search for such states in experimental searches for rare matter.

Of further interests are the exotic states which appear specifically when the symmetry is broken directly to the Standard Model. The "Wilsonian" sectors then contain also states which carry the Standard Model charges, but with fractional charge under the $U(1)_{Z^{\prime}}$ symmetry. We have shown, in a specific model, that these states can, in fact, be stable. This is an exciting observation, for then the stable "Wilsonian" matter states can be natural dark matter candidates. Furthermore, their stability arises due to a well motivated local discrete symmetry [39].

The superstring derived standard-like models give rise to "Wilsonian" color triplets, electroweak doublets with the standard charge assignment, and to Standard Model singlets. Of those the Standard Model singlets are the most suited to be the dark matter.

The appearance of a good dark matter candidate in the superstring derived standard-like models provides further motivation to focus on the phenomenology of this class of models. Although our analysis and results are limited to the models in the free fermionic formulation, the mechanism which gives rise to the "Wilsonian" matter states is generic in superstring models. Thus, exotic "Wilsonian" matter states may be the generic, long-sought, signature of string unification. It is of further interest to study other phenomenological properties of these states. Such questions are currently under investigation and will be reported in future publications.

\section{Acknowledgments}

We thank R. Field, D. Kennedy, D. Lichtenberg, R. Oakes, P. Ramond, P. Sikivie and C. Thorn for discussions. Special thanks to K. Dienes for comments on the manuscript. AEF thanks the Institute for Advanced Study for its hospitality while part of this work was conducted. CC thanks E. Herman, L. Gordon, A. White and the Theory group at Argonne for hospitality. This work was supported in part by DOE Grant No. DE-FG-0586ER40272 and by KOSEF. 


\section{Appendix A. The Mass splitting}

In this appendix we comment on the mass splitting relation between the two heavy-light mesons $U_{0}$ and $U_{-1}$. The goal of our discussion here is to show in a semi-quantitative way that the mass splitting between these two heavy-light mesons is compatible with zero and even with a $U_{0}$ lighter than $U_{1}$.

While transition and elastic form factors are calculable in the heavy quark effective theory, the calculation of the static properties of hadrons, such as the electromagnetic mass splitting of mesons containing heavy-light quarks, relies on drastic approximations with a built-in model dependence.

However, a simple estimate of the mass splitting between the two states $U_{0}(\bar{D} d)$ and $U_{-1}(D \bar{u})$ can be obtained in the context of potential models within a nonrelativistic approximation. Although this may not look appropriate, it has been shown that, even in the context of light mesons, the non-relativistic approximation to the analysis of the spectra of these systems (which are relativistic) has been quite successful when a Coulomb + linear + constant potential has been used to model the interaction. On the other hand, relativistic approaches based on bag models 54 have also produced satisfactory results. In ref. [55] it has been argued that there is an interesting duality relation between the bag model solutions (with a quadratic potential) and those produced by non-relativistic potential models. This observation seems to clarify, to some extent, that the non-relativistic approach is in part consistent even for systems which are intrinsically relativistic [59]. With these motivations in mind, let us first analyze the electromagnetic (plus mass difference of the two constituent quarks) contributions to the mass splitting. The interaction may be taken to be Coulomb (at short distances) + linear (the confining tail). We may assume that, in a meson, when one of the two quarks gets very heavy, the bound state is hydrogen-like. If we neglect the effect of the strong interactions (which is a questionable assumption since $\alpha_{s}$ is not small at distances of $1 \mathrm{fm}$ ) the potential of the Breit-Fermi Hamiltonian corresponding to the electromagnetic interaction, for states of with zero angular momentum is given by

$$
V_{e m}=\alpha_{e m} \frac{Q_{1} Q_{2}}{r}-\alpha_{e m} \frac{8 \pi}{3} \frac{\mathbf{S}_{1} \cdot \mathbf{S}_{\mathbf{2}}}{m_{1} m_{2}} \delta^{(3)}(\mathbf{r}) .
$$

$m_{1}$ and $m_{2}$ are the masses of the two quarks and $Q_{i}$ the corresponding electromagnetic charges. For spin singlet configurations

$$
\langle V\rangle_{0^{-}}=\alpha_{e m}\left\langle Q_{1} Q_{2}\right\rangle\left(\left\langle\frac{1}{r}\right\rangle+\frac{1}{m_{1} m_{2}}|R(0)|^{2}\right),
$$

with $R(r)$ the radial wave function of the ground state $(\psi(r)=(1 / \sqrt{4 \pi}) R(r))$. The spin interactions drops out in the heavy mass limit of any of the two quarks. Under these assumptions we get

$$
M_{U_{0}}-M_{U_{-1}}=\left(m_{d}-m_{u}\right)-\frac{\alpha_{e m}}{3}\left\langle\frac{1}{r}\right\rangle .
$$


If we use the estimate $m_{d}-m_{u} \approx M_{K^{0}}-M_{K^{+}}=4 \mathrm{MeV}$ for the constituent mass of the two light quarks [56] and a size of the bound state of $1 \mathrm{fm}$, then we get that the electromagnetic corrections are $1 \mathrm{MeV}$, thereby giving a positive $M_{U_{0}}-M_{U_{-1}} \approx 3$ $\mathrm{MeV}$. This difference can be reduced by strong interaction effects which can provide a $\Delta M_{s}$ which can even over-compensate $\Delta M_{e m}$ [56]. In fact, the difference between the up and the down quark masses causes a difference between the expectation values of the strong interaction Breit-Fermi Hamiltonian. This effect, therefore, can be responsible of a reduction of the mass splitting between the two states. We remark here that, in the case of neutral and charged B mesons, all these arguments do apply. The particle data book [51] gives a mass splitting between $B^{0}$ and $B^{-}$which is compatible with zero.

Let us now comment on the estimate of the electromagnetic interaction to the mass splitting of heavy-light mesons in the context of the heavy quark effective theory. At the same time we will also comment on the strong interaction contribution to the splitting which plays a significant role -but is still not calculable- in the context of these same effective theories. Unfortunately, the evaluation of the binding energy of the light degrees of freedom from the heavy quark theory suffers from a large uncertainty. The discussion which follows has been included to make this point more explicit.

In the heavy quark limit, the interaction between the quark spin and the chromomagnetic field vanishes, being inversely proportional to the heavy quark mass $m_{Q}$. Beside the spin symmetry, a new symmetry appears in the effective description of the bound state, since the interaction between the heavy and the light degrees of freedom $(u, d)$ is flavor blind. We picture the heavy quark then acting as a static color force. There are two basic scales appearing in the effective theory: $m_{Q}$ and $\bar{\Lambda} / m_{Q} \equiv\left(m_{\text {hadron }}-m_{Q}\right) / m_{Q}$, with $\bar{\Lambda}$ the scale of the light degrees of freedom. While $m_{Q}$ sets the scale for the perturbative expansion (in $\alpha_{s}\left(m_{Q}\right)$ ) of the theory, the second scale characterizes the nonperturbative contributions of additional form factors in the form of matrix elements of higher dimensional operators. Notice that the QED analogy breaks down exactly due to the presence of this second scale. To leading order in $1 / m_{Q}, \bar{\Lambda}=m_{\text {hadron }}-m_{Q}$ is flavor and spin independent. From QCD sum rules, using the pole mass for $m_{Q}$ (and neglecting renormalon ambiguities (see [58 and refs. therein) one gets the estimate

$$
\bar{\Lambda}=570 \pm 70 \mathrm{MeV}
$$

(see [58 and refs. therein). The uncertainty in Eq. (A.4) largely over compensates the electromagnetic contribution to the splitting.

Let us now comment briefly on the electromagnetic breaking contributions as estimated in the context of the heavy quark effective theory in ref. [57]. The calculation of the electromagnetic splitting has been based, in the past, on the use of dispersion relations for forward Compton scattering [60]. We remark that the study of factorization in exclusive processes [62 [61] and of dispersion relations, in particular for 
fixed angle Compton scattering [63], has a long and involved history. In the forward region, where perturbative QCD breaks down, the treatment of the process suffers from a strong model dependence. Therefore, the use of dispersion relations at very low momentum transfer, as needed in the case of the calculation of mass splittings, is an uncharted territory. The basic strategy of the method in [57] is to relate the order $e^{2}$ isospin breaking corrections to the forward Compton scattering amplitude $T$ and then use the heavy quark effective theory in the large $N_{c}$ limit to write $T$ in terms of heavy meson form factors.

Neglecting $1 / m_{q}^{2}$ corrections, the authors of ref. [57] give

$$
M_{U^{+}}-M_{U^{0}} \sim+1.7-0.13\left(\frac{\beta}{1 \mathrm{GeV}^{-1}}\right)-0.03\left(\frac{\beta}{1 \mathrm{GeV}^{-1}}\right)^{2},
$$

where $\beta \sim 1 / m_{Q}$ measures the matrix element of the decay of the first excited heavy meson state into the ground state plus a photon.

The use of large $N_{c}$ arguments and the ansatze used to match the dimensional counting behavior of the heavy quark form factor introduce a model dependence into the theory. The authors of ref. [57] argue that their results are accurate within $30 \%$. Notice that this result is compatible with the naive estimate obtained from the quark model discussion we have presented above. Also, notice that the combination of this result with Eq. (A.4), in particular the large uncertainty in the estimate of $\Lambda$ tells us that $M_{U_{0}}-M_{U_{-1}}$ is not incompatible with zero, as suggested by potential model calculations.

\section{Appendix B. Quark Decay Modes}

In this appendix we summarize the derivation of the Feynman rules for $N=1$ supersymmetric QCD and define our conventions for the various diagrams.

The Lagrangian is defined by [64

$$
\mathcal{L}=\bar{q} i \not D q-\frac{1}{4} F^{\mu \nu} F_{\mu \nu}+\left(D^{\mu} \tilde{q}\right)^{*} D_{\mu} \tilde{q}
$$

where

$$
\not D_{i j}=\not \delta_{i j}-\frac{i g}{2} \not A_{a} T_{i j}^{a}
$$

In SUSY QCD there is a gluino-gluino-gluon term,

$$
\mathcal{L}_{g \tilde{g} \tilde{g}}=\frac{i}{2} g_{s} f_{a b c} \overline{\tilde{g}}_{a} \gamma_{\mu} \tilde{g}_{b} A_{c}^{\mu}
$$

quark-squark-gluino term.

$$
\mathcal{L}_{q \tilde{q} \tilde{g}}=-\sqrt{2} g_{s} T_{j k}^{a} \sum_{i=u, d}\left(\overline{\tilde{g}}_{a} P_{L} q_{i}^{k} \tilde{q}_{i L}^{j *}+\bar{q}_{i}^{j} P_{R} \tilde{g}_{a} \tilde{q}_{i L}^{k}-\overline{\tilde{g}}_{a} P_{R} q_{i}^{k} \tilde{q}_{i R}^{j *}-\bar{q}_{i}^{j} P_{L} \tilde{g}_{a} \tilde{q}_{i R}^{k}\right),
$$




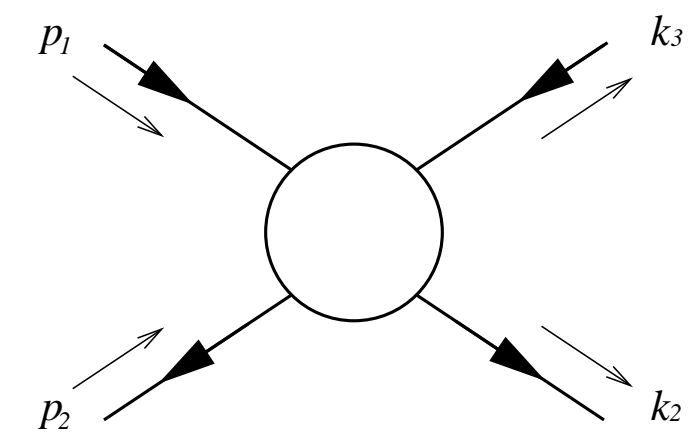

Figure 5: Momentum and fermion number flow

where $i$ is summed over the three families of quarks and $T^{a}=\lambda^{a} / 2$ are the usual $S U(3)$ generators. The two chirality projectors are defined by $P_{L}=\left(1-\gamma_{5}\right) / 2$ and $P_{R}=\left(1+\gamma_{5}\right) / 2$. The generators of $S U(3)$ are taken to be hermitian. The quarkgluon vertex, in these conventions, is given by

$$
\mathcal{L} q \bar{q} g=-\bar{q} A_{\mu}^{a} \gamma^{\mu} q T^{a} .
$$

In momentum space we get $-i g_{s} \gamma_{\mu} T^{a}$ for the $q \bar{q} g$ vertex and $-g_{s} f_{a b c} \gamma_{\mu}$ for the $\tilde{g} \tilde{g} g$ vertex.

We introduce the following expansions for the Dirac fermions (quarks), Majorana fermions (gluinos) and for the scalar quarks

$$
\begin{aligned}
& q(x)=\int \frac{d^{3} k}{(2 \pi)^{3}}\left(\frac{m}{k_{0}}\right)\left(b(k) u(k) e^{-i k x}+d^{\dagger}(k) v(k) e^{i k x}\right) \\
& \tilde{g}(x)=\int \frac{d^{3} k}{(2 \pi)^{3}}\left(\frac{m}{k_{0}}\right)\left(\tilde{b}(k) \tilde{u}(k) e^{-i k x}+\tilde{b}^{\dagger}(k) \tilde{v}(k) e^{i k x}\right) \\
& \tilde{q}(x)=\int \frac{d^{3} k}{(2 \pi)^{3} 2 \omega_{k}}\left(a(k) e^{-i k x}+b^{\dagger}(k) e^{i k x}\right) .
\end{aligned}
$$

Notice that in (B.6) (B.7) and (B.8) we have omitted the sum over the polarizations for simplicity. We enforce the usual quantization conditions

$$
\begin{aligned}
& \left\{b(k), b^{\dagger}\left(k^{\prime}\right)\right\}=(2 \pi)^{3}\left(\frac{k_{0}}{m}\right) \delta^{3}\left(\mathbf{k}-\mathbf{k}^{\prime}\right) \\
& \left\{\tilde{b}(k), \tilde{b}^{\dagger}\left(k^{\prime}\right)\right\}=(2 \pi)^{3}\left(\frac{k_{0}}{m}\right) \delta^{3}\left(\mathbf{k}-\mathbf{k}^{\prime}\right) ; \\
& \left\{d(k), d^{\dagger}\left(k^{\prime}\right)\right\}=(2 \pi)^{3}\left(\frac{k_{0}}{m}\right) \delta^{3}\left(\mathbf{k}-\mathbf{k}^{\prime}\right) .
\end{aligned}
$$

Scattering amplitudes can be defined in terms of the vacuum-to-vacuum correlators either by using the LSZ reduction formula or, more simply, by calculating 

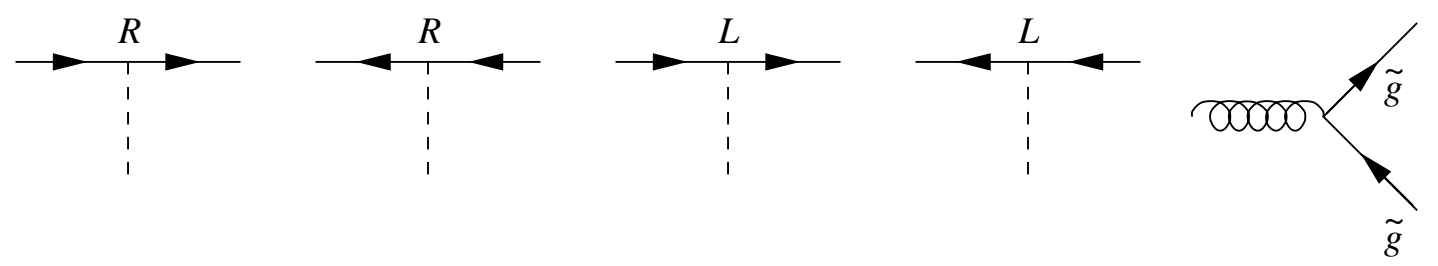

Figure 6: Gluino vertices

expectation values of the interaction terms in initial and final states.

$$
S_{f i}=\frac{\left(i g_{s}\right)^{2}}{2} \int d^{4} y_{1} d^{4} y_{2}\left\langle Q \bar{Q}\left|\mathcal{L}\left(y_{1}\right) \mathcal{L}\left(y_{2}\right)\right| \tilde{g} \tilde{g}\right\rangle .
$$

We have defined

$$
|Q \bar{Q}\rangle \equiv b^{\dagger}\left(p_{1}\right) d^{+}\left(p_{2}\right)|0\rangle ; \quad|\tilde{g} \tilde{g}\rangle=\tilde{b}^{\dagger}\left(k_{3}\right) \tilde{b}^{\dagger}\left(k_{2}\right)|0\rangle .
$$

Notice that it is convenient to fix a specific convention by labeling one of the two gluinos as first particle $\left(k_{2}\right)$ and the second one as second particle $\left(k_{3}\right)$. Define $(A B)_{c} \equiv\langle 0|A B| 0\rangle$ to be the usual Wick contraction and set (symbolically)

$$
\begin{aligned}
& q(x) \sim b u+d^{\dagger} \bar{v} \\
& \bar{q}(x) \sim b^{\dagger} \bar{u}+d \bar{v} \\
& \tilde{g}(x) \sim \tilde{b} u+\tilde{b}^{\dagger} \tilde{v} \\
& \overline{\tilde{g}}(x) \\
& \tilde{q}(x) \tilde{b}^{\dagger} \tilde{u}+\tilde{b} \tilde{\tilde{v}} \\
& \tilde{q}\left(a+a^{\dagger},\right.
\end{aligned}
$$

in order to get the only non-vanishing contractions with the initial (final) states

$$
\begin{aligned}
& \left(q(x) b^{\dagger}(k)\right)_{c}=u(k) e^{-i k x} \\
& \left(\bar{q}(x) d^{\dagger}(k)\right)_{c}=\bar{v}(k) e^{-i k x} \\
& \left(\tilde{g}(x) \tilde{b}^{\dagger}(k)\right)_{c}=\tilde{u}(k) e^{-i k x} \\
& \left(\overline{\tilde{g}}(x) \tilde{b}^{\dagger}(k)\right)_{c}=\overline{\tilde{v}}(k) e^{-i k x} \\
& (b(k) \bar{g}(x))_{c}=\overline{\tilde{u}}(k) e^{i k x} \\
& (b(k) \tilde{g}(x))_{c}=\tilde{v}(k) e^{-i k x} .
\end{aligned}
$$

There are 5 diagrams which contribute in the supersymmetric case to the annihilation of a $Q \bar{Q}$ pair into two gluinos. In our calculation we neglect mixing between the squark fields. The amplitudes are given by

$$
A_{1}=-\frac{2 i \alpha_{s}}{t-M_{s}^{2}}\left(T^{b} T^{a}\right)_{j k} \overline{\tilde{u}}^{a}\left(k_{3}\right) P_{R} u^{k}\left(p_{1}\right) \bar{v}^{j}\left(p_{2}\right) P_{L} \tilde{v}^{b}\left(k_{2}\right)
$$




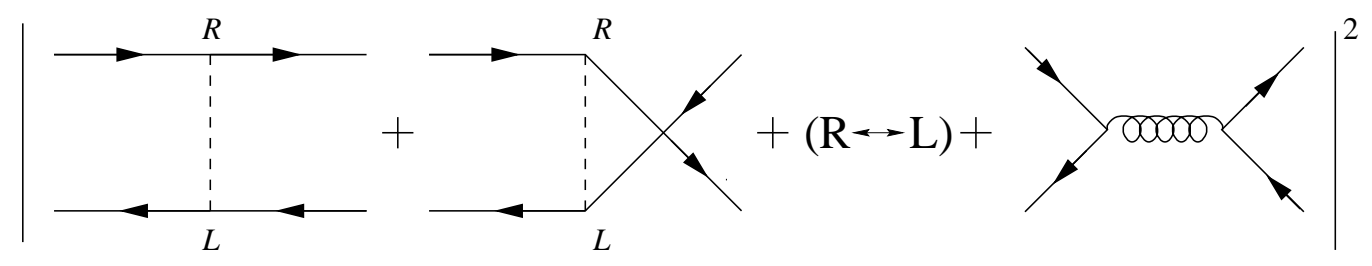

Figure 7: $Q \bar{Q} \longrightarrow \tilde{g} \tilde{g}$ annihilation

$$
\begin{aligned}
& A_{2}=\frac{2 i \alpha_{s}}{t-M_{s}^{2}}\left(T^{b} T^{a}\right)_{j k} \overline{\tilde{u}}^{a}\left(k_{2}\right) P_{R} u^{k}\left(p_{1}\right) \bar{v}^{j}\left(p_{2}\right) P_{L} \tilde{v}^{b}\left(k_{3}\right) \\
& A_{3}=-\frac{2 i \alpha_{s}}{t-M_{s}^{2}}\left(T^{b} T^{a}\right)_{j k} \overline{\tilde{u}}^{a}\left(k_{3}\right) P_{L} u^{k}\left(p_{1}\right) \bar{v}^{j}\left(p_{2}\right) P_{R} \tilde{v}^{b}\left(k_{2}\right) \\
& A_{4}=\frac{2 i \alpha_{s}}{t-M_{s}^{2}}\left(T^{b} T^{a}\right)_{j k} \overline{\tilde{u}}^{a}\left(k_{2}\right) P_{L} u^{k}\left(p_{1}\right) \bar{v}^{j}\left(p_{2}\right) P_{R} \tilde{v}^{b}\left(k_{3}\right) \\
& \left.A_{5}=-\frac{1}{2 s} \alpha_{s} T_{j k}^{d} f^{d a b} \overline{\tilde{u}}^{a}\left(k_{3}\right) \gamma^{\mu} \tilde{v}^{k}\left(k_{2}\right)\right) \bar{v}^{j}\left(p_{2}\right) \gamma_{\mu} u^{k}\left(p_{1}\right)
\end{aligned}
$$

where we have set $s=\left(p_{1}+p_{2}\right)^{2}$ and $t=\left(p_{1}-k_{3}\right)^{2}$. Here $a$ and $b$ are SU(3) octet indices for the two gluino spinors $\tilde{v}$ and $\tilde{u}$, while $j$ and $k$ are the color indices in the fundamental. We have assumed that the left and right squarks have equal masses $\left(M_{s}\right)$. We also take the final gluinos to be massless. Notice that in the case of annihilation into a quark-antiquark pair, the number of diagrams is doubled (with a factor of two also for the $A_{5}$, absent in the QCD case) because of additional non-zero contractions with the Majorana final states. Notice that in the direct and exchange diagrams we have distinguished between the fermion flow and the momentum flow. In each exchanged $t$-channel diagram the fermion flow for the final states is reversed compared to the flow for each direct diagram.

The averaged cross section for the annihilation of the uniton into two gluinos or into two gluons is generically defined by

$$
\left\langle\left|\mathcal{M}_{U}\right|^{2}\right\rangle=\frac{1}{(3)^{2}(2)^{2}}\left|\mathcal{M}_{U}\right|^{2}
$$

where we have averaged over spin and color. The calculation has been performed in the most general case, taking all the mass parameters to be different. The final result is too cumbersome to be given here.

For the annihilation of two heavy quarks into two gluinos in the equal-mass limit we get

$$
|\mathcal{M}|^{2}=\frac{256 \pi^{2} \alpha_{s}^{2}}{27 s^{2}\left(t-m^{2}\right)\left(s+t-m^{2}\right)}\left(9 m^{8}-18 m^{6} s+13 m^{4} s^{2}-8 m^{2} s^{3}\right.
$$




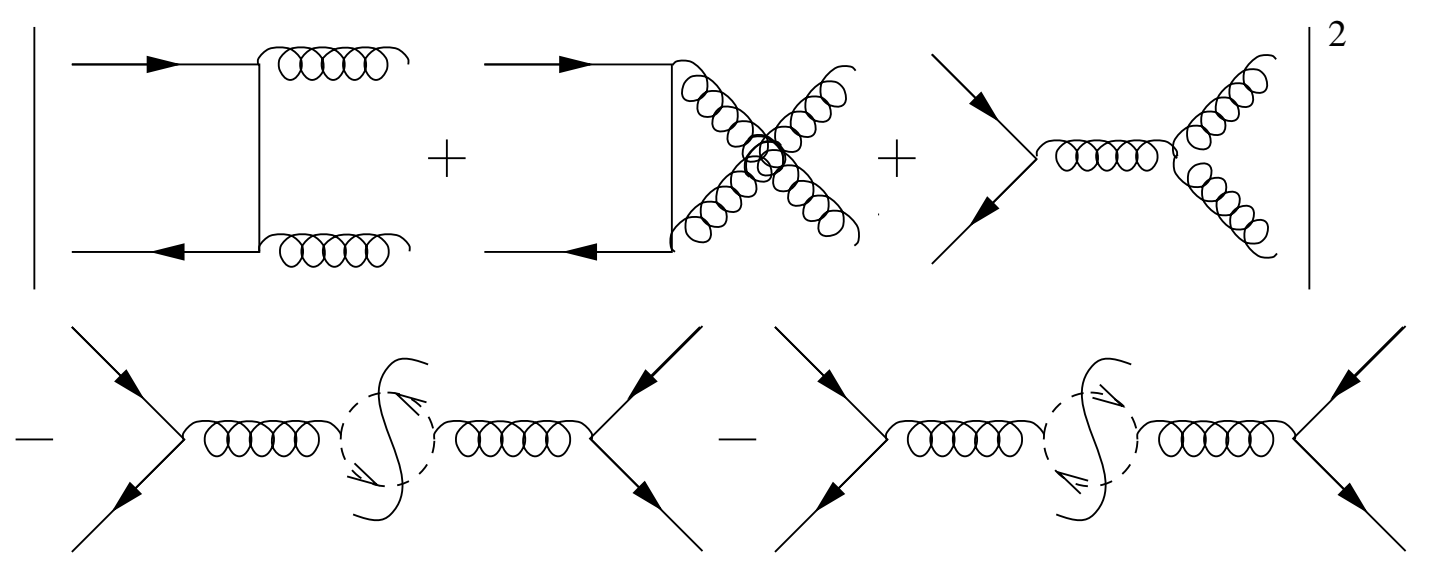

Figure 8: $Q \bar{Q} \longrightarrow g g$ annihilation

$$
\begin{aligned}
& +\left(13 s^{3}-44 m^{2} s^{2}+63 m^{4} s-36 m^{6}\right) t \\
& \left.+\left(31 s^{2}-72 m^{2} s+54 m^{4}\right) t^{2}+\left(27 s-36 m^{2} t^{3}\right) t^{3}+9 t^{4}\right) .
\end{aligned}
$$

The total cross section is

$$
\sigma=\frac{16 \pi \alpha_{s}^{2}}{27 A^{3}}\left(-24 m^{4}-22 m^{2} s+7 s^{2}-m^{2} \log \left(\frac{s+A}{s-A}\right)\right) .
$$

We have set $A=\sqrt{s\left(s-4 m^{2}\right)}$. In the non-relativistic limit we get $s=4 m^{2}\left(1+v_{c m}^{2}\right)$ and $A=4 m^{2} v_{c m}$ with a total cross section

$$
\sigma|v|=\frac{64 \pi \alpha_{s}^{2}}{27 m^{2}}+\mathrm{O}\left(v^{2}\right)
$$

where $|v|=2\left|v_{c m}\right|$

Let us now comment on the $Q \bar{Q}$ annihilation into two gluons. The calculation is performed in the Feynman gauge and we have included the ghost contribution diagram (since there are two gluons in the final state) to remove the unphysical polarizations. We recall that in the case of massive quarks all the interference diagrams give a non-vanishing contribution.

We give expressions for all the cross sections in the relativistic and in the nonrelativistic cases. The total cross section is given by

$$
\sigma=\frac{16 \pi \alpha_{s}^{2}}{27 m^{2} s^{2} A^{2}}\left(-\left(7 s+31 m^{2}\right) A+4\left(s^{2}+4 m^{2} s+m^{4}\right) \log \left(\frac{s+A}{s-A}\right)\right) .
$$

In the non-relativistic limit we get

$$
\sigma|v|=\frac{14 \pi \alpha_{s}^{2}}{27 m^{2}}+\mathrm{O}\left(v^{2}\right) .
$$




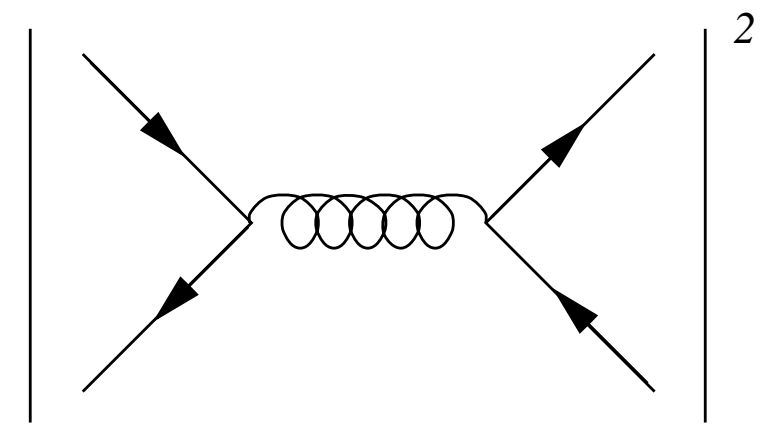

Figure 9: $Q \bar{Q} \longrightarrow q \bar{q}$ annihilation

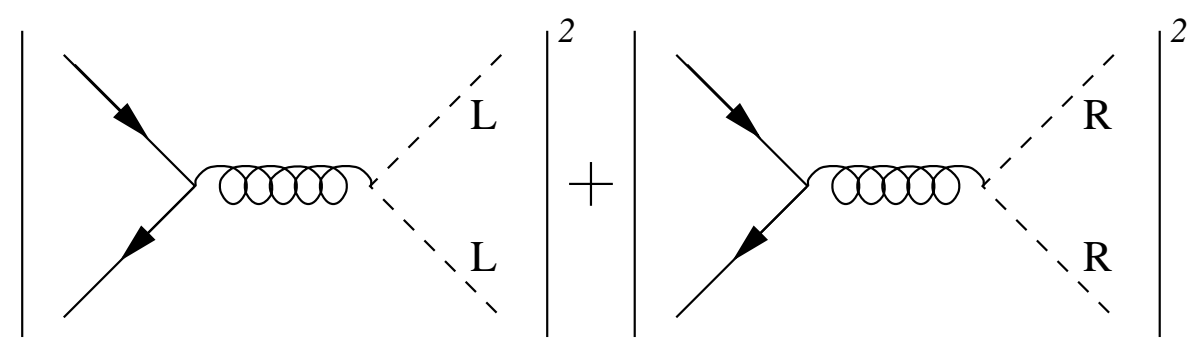

Figure 10: $Q \bar{Q} \longrightarrow \tilde{q} \tilde{q}^{*}$ annihilation

In the case of the annihilation of a heavy $Q \bar{Q}$ pair into a light $q \bar{q}$ pair we get

$$
\sigma=\frac{8 \alpha_{s}^{2} \pi\left(s+2 m^{2}\right)}{27 s \sqrt{s\left(s-4 m^{2}\right)}}
$$

which in the non-relativistic limit gives

$$
\sigma|v|=\frac{2 \pi \alpha_{s}^{2}}{9 m^{2}}+\mathrm{O}\left(v^{2}\right)
$$

If we sum over all 6 quark flavors of the final state we get

$$
\sigma|v|=\frac{4 \pi \alpha_{s}^{2}}{3 m^{2}}+\mathrm{O}\left(v^{2}\right)
$$

The total cross section for squark production from $Q \bar{Q}$ annihilation is given by

$$
\begin{aligned}
\sigma & =\frac{4 \alpha_{s}^{2} \pi\left(s+2 m^{2}\right)}{27 s \sqrt{s\left(s-4 m^{2}\right)}} \\
& =\frac{4 \alpha_{s}^{2} \pi\left(s+2 m^{2}\right)}{27 s A} .
\end{aligned}
$$


In the non-relativistic limit we get

$$
\sigma|v|=\frac{\pi \alpha_{s}^{2}}{9 m^{2}}+\mathrm{O}\left(v^{2}\right)
$$

If we sum over all 6 flavors of the quarks in the final state we get

$$
\sigma|v|=\frac{2 \pi \alpha_{s}^{2}}{3 m^{2}}+\mathrm{O}\left(v^{2}\right)
$$




\section{References}

[1] For a review, see:

M.B. Green, J.H. Schwarz, and E. Witten, Superstring Theory, Vols. 1 \& 2 (Cambridge University Press, Cambridge, 1987).

[2] D.J. Gross, J.A. Harvey, J.A. Martinec, and R. Rohm, Phys. Rev. Lett. 54 (1985) 502; Nucl. Phys. B256 (1986) 253.

[3] P. Candelas, G.T. Horowitz, A. Strominger, and E. Witten, Nucl. Phys. B258 (1985) 46.

[4] D.C. Lewellen, Nucl. Phys. B337 (1990) 61;

J. Ellis, J.L. Lopez and D.V. Nanopoulos, Phys. Lett. B245 (1990) 375;

A. Font, L.E. Ibáñez, and F. Quevedo, Nucl. Phys. B345 (1990) 389;

S. Chaudhuri, S.-W. Chung, G. Hockney, and J. Lykken, hep-ph/9501361;

G. Aldazabal, A. Font, L.E. Ibáñez, and A.M. Uranga, hep-th/9410206;

G. Cleaver, hep-th/9506006;

D. Finnell, hep-th/9508073;

J. Erler, hep-th/9602032;

K. Dienes and J. March-Russell, hep-th/9604112.

[5] M. Dine, el al., Nucl. Phys. B259 (1985) 549;

B. Greene, K.H. Kirklin, P.J. Miron and G.G. Ross , Phys. Lett. B180 (1986) 69; Nucl. Phys. B292 (1987) 606;

R. Arnowitt and P. Nath, Phys. Rev. D39 (1989) 2006; D42 (1990) 2498; Phys. Rev. Lett. 62 (1989) 222.

[6] I. Antoniadis, J. Ellis, J. Hagelin, and D.V. Nanopoulos, Phys. Lett. B231 (1989) 65.

[7] I. Antoniadis, G.K. Leontaris, and J. Rizos, Phys. Lett. B245 (1990) 161;

G.K. Leontaris, hep-ph/9601337.

[8] T.T. Burwick, A.K. Kaiser and H.F. Muller Nucl. Phys. B362 (1991) 232;

A. Kagan and S. Samuel, Phys. Lett. B284 (1992) 289.

[9] J. Lopez, D.V. Nanopoulos, and K. Yuan, Nucl. Phys. B399 (1993) 654, hepth/9203025.

[10] L.E. Ibañez, J.E. Kim, H.P. Nilles and F. Quevedo, Phys. Lett. B191 (1987) 282

A. Font, L.E. Ibanez, H.P. Nilles and F. Quevedo, Phys. Lett. B210 (1988) 101;

A. Font, L.E. Ibanez, F. Quevedo and A. Sierra, Nucl.Phys. B331 (1990) 421;

D. Bailin, A. Love and S. Thomas, Nucl. Phys. B298 (1988) 75; 
J.A. Casas, E.K. Katehou and C. Muñoz, Nucl. Phys. B317 (1989) 171;

S. Chaudhuri, G. Hockney, and J. Lykken, hep-th/9510241.

[11] A.E. Faraggi, D.V. Nanopoulos and K. Yuan, Nucl. Phys. B335 (1990) 347.

[12] A.E. Faraggi, Phys. Lett. B278 (1992) 131.

[13] A.E. Faraggi, Nucl. Phys. B387 (1992) 239, hep-th/9208024.

[14] A.E. Faraggi, Phys. Lett. B339 (1994) 223, hep-ph/9408333.

[15] E. Witten, Nucl. Phys. B258 (1985) 75;

J.D. Breit, B.A. Ovrut, and G.C. Segré, Phys. Lett. B158 (1985) 75;

A. Sen, Phys. Rev. Lett. 55 (1985) 33.

[16] A.E. Faraggi, Nucl. Phys. B428 (1994) 111, hep-ph/9403312.

[17] T. Banks, D. Kaplan and A. Nelson, Phys. Rev. D49 (1994) 779.

[18] E. Halyo, Nucl. Phys. B438 (1995) 138.

[19] X.G. Wen and E. Witten, Nucl. Phys. B261 (1985) 651;

G.G. Athanasiu, J.J. Atick, Michael Dine and Willy Fischler, Phys. Lett. B214 (1988) 55;

A. Schellekens, Phys. Lett. B237 (1990) 363.

[20] J. Ellis, J.L. Lopez and D.V. Nanopoulos, Phys. Lett. B247 (1990) 257.

[21] P. Huet, Nucl. Phys. B350 (1991) 375.

[22] A.E. Faraggi, Phys. Rev. D46 (1992) 3204.

[23] A.E. Faraggi, Phys. Lett. B274 (1992) 47.

[24] S. Chang, C. Corianò and A.E. Faraggi, hep-ph/9603272.

[25] K.R. Dienes and A.E. Faraggi, Phys. Rev. Lett. 75 (1995) 2646, hep-th/9505018;

Nucl. Phys. B457 (1995) 409, hep-th/9505046.

[26] A.E. Faraggi, Nucl. Phys. B407 (1993) 57, hep-ph/9210256; Phys. Lett. B326 (1994) 62, hep-ph/9311312.

[27] A.E. Faraggi, Phys. Lett. B302 (1993) 202, hep-ph/9301268.

[28] S. Kalara, J. Lopez, and D.V. Nanopoulos, Nucl. Phys. B353 (1991) 650.

[29] A.E. Faraggi, Nucl. Phys. B403 (1993) 101, hep-th/9208023.

[30] M. Turner, astro-ph/9503017. 
[31] P. Ginsparg, Phys. Lett. B197 (1987) 139.

[32] M. Dine and N. Seiberg, Phys. Rev. Lett. 55 (1985) 366;

V.S. Kaplunovsky, Phys. Rev. Lett. 55 (1985) 1036.

[33] V.S. Kaplunovsky, Nucl. Phys. B307 (1988) 145; Erratum: ibid. B382 (1992) 436, hep-th/9205070.

[34] U. Amaldi et al, Phys. Rev. D36 (1987) 1385;

P. Langacker and M. Luo, Phys. Rev. D44 (1991) 817;

J. Ellis, S. Kelley, and D. V. Nanopoulos, Phys. Lett. B260 (1991) 131;

U. Amaldi, W. de Boer, and H. Füstenau, Phys. Lett. B260 (1991) 447;

H. Arason et al, Phys. Rev. D46 (1992) 3945;

F. Anselmo, L. Cifarelli, A. Peterman, and A. Zichichi, Nuovo Cimento 105A (1992) 1179 ;

L. Langacker and N. Polonsky, Phys. Rev. D47 (1993) 4028, hep-ph/9210235;

A.E. Faraggi and B. Grinstein, Nucl. Phys. B422 (1994) 3, hep-ph/9308329.

[35] See, e.g., the following papers and references therein:

L.J. Dixon, V.S. Kaplunovsky, and J. Louis, Nucl. Phys. B355 (1991) 649;

I. Antoniadis, J. Ellis, R. Lacaze, D.V. Nanopoulos , Phys. Lett. B268 (1991) 188 ;

I. Antoniadis, K.S. Narain, and T.R. Taylor, Phys. Lett. B267 (1991) 37;

J.P. Derendinger, S. Ferrara, C. Kounnas and F. Zwirner, Nucl. Phys. B372 (1992) 145;

G. Lopes Cardoso and B.A. Ovrut, Nucl. Phys. B369 (1992) 351;

P. Mayr and S. Stieberger, Nucl. Phys. B412 (1994) 502, hep-th/9304055;

D. Bailin and A. Love, Phys. Lett. B292 (1992) 315;

D. Bailin, A. Love, W.A. Sabra, and S. Thomas, Mod. Phys. Lett. A10 (1995) 337, hep-th/9407049;

M. Chemtob, hep-th/9506018;

E. Kiritsis and C. Kounnas, Nucl. Phys. B442 (1995) 472, hep-th/9501020.

[36] I . Antoniadis, J. Ellis, S. Kelley and D.V. Nanopoulos, Phys. Lett. B272 (1991) 31

S. Kelley, J. Lopez, and D.V. Nanopoulos, Phys. Lett. B278 (1992) 140;

I. Antoniadis, G.K. Leontaris, and N.D. Tracas, Phys. Lett. B279 (1992) 58;

M.K. Gaillard and R. Xiu, Phys. Lett. B296 (1992) 71, hep-ph/9206206;

I. Antoniadis and K. Benakli, Phys. Lett. B295 (1992) 219, hep-th/9209020;

S.P. Martin and P. Ramond, Phys. Rev. D51 (1995) 6515, hep-ph/9501244.

[37] J.A. Casas and C. Munoz, Phys. Lett. B214 (1988) 543;

L.E. Ibáñez, Phys. Lett. B318 (1993) 73, hep-ph/9308365; 
K.R. Dienes, A.E. Faraggi, and J. March-Russell, Nucl. Phys. B467 (1996) 44, hep-th/9510223.

[38] For an alternative proposal based on strongly interacting strings see E. Witten, hep-th/9602070.

[39] L.M. Krauss and F. Wilczek, Phys. Rev. Lett. 62 (1989) 1221.

[40] A.E. Faraggi and E. Halyo, Phys. Lett. B307 (1993) 305, hep-ph/9301261; Nucl. Phys. B416 (1994) 63, hep-ph/9306235.

[41] M. Dine, N. Seiberg, and E. Witten, Nucl. Phys. B289 (1987) 589;

J.J. Atick, L.J. Dixon, and A. Sen, Nucl. Phys. B292 (1987) 109;

S. Cecotti, S. Ferrara, and M. Villasante, Int. J. Mod. Phys. A2 (1987) 1839.

[42] J.C. Pati and A. Salam, Phys. Rev. D8 (1973) 1240; Phys. Rev. Lett. 31 (1973) 661; Phys. Rev. D10 (1974) 275.

[43] M. Srednicki, R. Watkins and K. Olive, Nucl. Phys. B310 (1988) 693.

[44] E.W. Kolb and M.S. Turner, The Early Universe (Addison-Wesley, New York, 1990).

[45] A. Gould, B. Draine, R. Romani and S. Nussinov, Phys. Lett. B238 (1990) 337.

[46] M. W. Goodman and E. Witten, Phys. Rev. D31 (1985) 3059.

[47] D.B. Sanders,E. S. Phinney, G. Neugebauer, B. T. Soifer and K. Mathews, Astrophys. J. 347, (1989) 29;

A. van Dalen and R. K. Schaefer, Astrophys. J. 398, (1992) 33;

A.N. Taylor and M. Rowan-Robinson, Nature 359, (1992) 396;

J.A. Holtzman and J.R. Primack, Astrophys. J. 405, (1993) 428.

[48] J.M. Bardeen, J. R. Bond and G. Efstathiou, Astrophys. J. 321, (1987) 28;

E. J. Chun, H. B. Kim and J. E. Kim, Phys. Rev. Lett. 72 (1994) 1956;

S. Chang and H.B. Kim, hep-ph/9604222.

[49] G. D. Starkman, A. Gould, R. Esmailzadeh and S. Dimopoulos, Phys. Rev. D41 (1990) 3594 .

[50] E. Nardi and E. Roulet, Phys. Lett. B245 (1990) 105.

[51] Particle Data Group, Review of Particle Properties, Phys. Rev. D50 (1994) 1, and 1995 off-year partial update for the 1996 edition available on the PDG WWW pages (URL: http://pdg.Ibl.gov/). 
[52] B. Holdom, Phys. Lett. B339, 114 (1994);

P. Chiappetta, J. Layssac, F.M. Renard and C. Verzegnassi, preprint PM/96-05, hep-ph/9601306;

G. Altarelli, N. Di Bartolomeo, F. Feruglio, R. Gatto and M. Mangano, hepph/9601324:

K.S. Babu, C. Kolda and J. March-Russell, hep-ph/9603212;

P.H. Frampton and B.D. Wright, hep-ph/9604260;

K. Agashe, M. Graesser, I. Hinchliffe and M. Suzuki, hep-ph/9604266

[53] A.E. Faraggi and M. Masip, hep-ph/9604302.

[54] A. Chodos, R.L. Jaffe, K. Johnson and C.B. Thorn, Phys. Rev. D10 (1974) 2599 .

[55] B. Rosenstein, Phys. Rev. D33 (1986) 813.

[56] W. Lucha, F. Schöberl and D. Gromes, Phys. Rep. 200 (1991) 127.

[57] M. Luty and R. Sundrum, Phys. Rev. D52 (1995) 1627.

[58] T. Mannel, hep-ph/9409387.

[59] D. Flamm, F.Schöberl and H. Uematsu, Nuovo Cimento 98A (87) 559.

[60] R.P. Feynman and G. Speisman, Phys. Rev. D94 (1954) 500;

M. Cini, E. Ferrari and R. Gatto, Phys. Rev. Lett. 2 (1959) 7;

A. Zee, Phys. Rep. 3 (1972) 129.

[61] J. Botts and G. Sterman, Nucl. Phys. B325 (1989) 526;

H. N. Li and G. Sterman, Nucl. Phys. B381 (1992) 129.

[62] G.P. Lepage and S.J. Brodsky, in Perturbative QCD, ed. A. H. Mueller (World Scientific, Singapore, 1989).

[63] C. Corianò, A. Radyushkin, and G. Sterman, Nucl. Phys. B405 (1993) 481;

C. Corianò, Nucl. Phys. B410 (1993) 481; Nucl. Phys. B434 (1995) 565;

C. Corianò and H. N. Li, Phys. Lett. B309 (1993) 481; Nucl. Phys. B434 (1995) 535 .

[64] H.E. Haber and G.L. Kane, Phys. Rep. 117 (1985) 75. 